\title{
An Adaptive Nonlinear Aircraft Maneuvering Envelope Estimation Approach for Online Applications
}

\author{
Stefan Schuet* \\ NASA Ames Research Center \\ Moffett Field, CA \\ Thomas Lombaerts ${ }^{\dagger}$ \\ German Aerospace Center DLR \\ Weßling, Germany \\ Diana Acosta* and Kevin Wheeler* and John Kaneshige*
NASA Ames Research Center, Moffett Field, CA
}

\begin{abstract}
A nonlinear aircraft model is presented and used to develop an overall unified approach to online trim and maneuverability envelope estimation with uncertainty quantification without any requirement for active input excitation. The concept of time scale separation makes this method suitable for the adaptive characterization of altered safe maneuvering limitations based on aircraft performance after impairment. The results can be used to provide pilot feedback and/or be combined with flight planning, trajectory generation, and guidance algorithms to help maintain safe aircraft operations in both nominal and off-nominal scenarios.
\end{abstract}

\section{Nomenclature}

$\mathbb{R}^{n} \quad$ Set of real $n$-vectors

$\mathbb{S}^{n}, \mathbb{S}_{++}^{n} \quad$ Set of $n \times n$ symmetric matrices, Set of $n \times n$ positive definite symmetric matrices

tr Trace operator, i.e., $\operatorname{tr} A$ is the sum of the diagonal elements of $A$

$|\cdot|,\|\cdot\| \quad$ Determinant, Euclidean norm for vector inputs

$V, \gamma, \chi \quad$ True airspeed, flight path angle, course angle

$F_{T} \quad$ Magnitude of net thrust force produced by engines

$F_{L}, F_{D}, F_{Y}$ Magnitude of lift, drag, and side forces

$f \quad$ Continuous dynamics model

$x, \mathbf{x} \quad$ State vector, Collection of state observations

$u, \mathbf{u} \quad$ Virtual input vector, Collection of virtual input observations

$\mathcal{B} \quad$ Set of allowable or achievable states and input vectors

$\tilde{x} \quad$ Average current and next state, i.e., $\tilde{x}(k)=[x(k+1)+x(k)] / 2$

$\alpha, \beta, \varphi \quad$ Angle of attack, Side-slip angle, Roll angle

$g, \mathrm{~m}, \mathrm{~S} \quad$ Acceleration due to gravity, Mass of the aircraft, Surface area of the aircraft wings

$\rho, \bar{q} \quad$ Air density, Dynamic pressure $\left(\bar{q}=\rho V^{2} / 2\right)$

$\mathcal{N}(\mu, \Sigma) \quad$ Multivariate normal distribution with mean $\mu$ and covariance matrix $\Sigma$.

$p(x \mid y) \quad$ Probability density function for the variable $x$ given $y$

$L_{i}, D_{j}, Y_{k} \quad$ Expansion coefficients for the non-dimensional coefficient of lift, drag, and side force

$c \quad$ Aerodynamic coefficient parameter vector, e.g., $c=\left[D_{0}, D_{1}, D_{2}, L_{0}, L_{1}, Y_{1}\right]$

\footnotetext{
${ }^{*}$ Researcher at NASA Ames Research Center, Intelligent Systems Division, AIAA Member, email: stefan.r.schuet@nasa.gov

${ }^{\dagger}$ Researcher at DLR, Institute of System Dynamics and Control, Department of Aircraft Dynamics, e-mail: thomas.lombaerts@dlr.de, currently Marie Curie Fellow and visiting researcher at NASA Ames Research Center, Intelligent Systems Division, Adaptive Control and Evolvable Systems (ACES), AIAA Member.
} 
$S \quad$ State process noise precision (a.k.a., inverse covariance) matrix

$c^{\star}, S^{\star} \quad$ Optimal estimates for $c$ and $S$

All units are SI.

\section{Introduction}

The safe maneuvering envelope is a fundamental property of the aircraft's design and overall current state of health. By definition it determines the overall capability of the aircraft. If actively monitored in time, it may function as an early warning system as well as provide anticipatory guidance to help avoid loss of control. For example, automated planning tools may use it to help pilots land safely under emergency landing conditions, ${ }^{1-3}$ or when combined with a display it may provide better pilot awareness of the aircraft state when an automation system switches off. Additionally, the physics-based maneuverability envelope can be analyzed separately from the control strategy, and knowledge of the envelope may for example unmask control limitations clouded by adaptive controllers, and even lower barriers to the introduction of more advanced unconventional control strategies. ${ }^{4}$ For these reasons, improved methods for tracking aircraft maneuverability in real-time may effectively help pilots avoid inappropriate crew response and further prevent or recover aircraft from upset conditions.

Generally, the maneuvering envelope is the set of safe aircraft state and control inputs. Unfortunately, because of the underlying nonlinear aircraft dynamics, this set is difficult to calculate accurately and rapidly enough to provide the pilot or automation system with reliable information in a diverse and rapidly changing environment. Previous research has considered a wide variety of approaches to meet the challenge. The most straight-forward of course is through wind tunnel testing, flight testing, and high-fidelity model-based computation. $^{5-8}$ A more sophisticated analysis is obtained by formulating the envelope estimation problem as a reachability problem, ${ }^{9}$ and then solving the associated Hamilton-Jacobi equations, often through the use of level set methods. ${ }^{10}$ Further extensions on this theme include leveraging the concepts of timescale separation ${ }^{11}$ or semi-Lagrangian level sets. ${ }^{12,13}$ Other alternatives rely on linearization and region of attraction analysis, ${ }^{14}$ quaternion-based control architectures,${ }^{15}$ robustness analysis,${ }^{16}$ frequency domain non-parametric system identification, ${ }^{17}$ or the Control-Centric Modeling methods suggested by Boeing. ${ }^{18}$ There even exist methods based on an artificial immune system paradigm. ${ }^{19-21}$

This article develops an integrated method complementary to much of the previous work on this topic. We begin by developing a simplified but effective nonlinear flight dynamics model in section II with important benefits including the ability to numerically calculate the corresponding trim envelope in real-time. The trim envelope however depends on aerodynamic parameters, and in section III we introduce a reliable Bayesian system identification technique, with a conservative approach to uncertainty quantification, tailored to the task of estimating the aerodynamic parameters from measured flight data. In section IV, we show how the dynamics model and trim envelope are combined to determine an extended safe maneuvering envelope, robust to the uncertainty established by the system identification process. Finally, main applications for the overall approach are summarized in section $\mathrm{V}$.

\section{Dynamics Model}

In this section, a focused approximate nonlinear model for the aircraft dynamics is presented. This model captures the slower aircraft dynamics, and ultimately enables the nonlinear analysis of maneuverability in flight path angle and total airspeed that is central to the discussion in this article.

Aerodynamic forces are most clearly organized around the velocity vector expressed using the kinematic axes, which are defined by the basis vectors

$$
\begin{aligned}
\hat{\boldsymbol{V}} & =\cos \gamma \cos \chi \hat{\boldsymbol{x}}_{E}+\cos \gamma \sin \chi \hat{\boldsymbol{y}}_{E}-\sin \gamma \hat{\boldsymbol{z}}_{E} \\
\hat{\gamma} & =-\sin \gamma \cos \chi \hat{\boldsymbol{x}}_{E}-\sin \gamma \sin \chi \hat{\boldsymbol{y}}_{E}-\cos \gamma \hat{\boldsymbol{z}}_{E} \\
\hat{\chi} & =-\sin \chi \hat{\boldsymbol{x}}_{E}+\cos \chi \hat{\boldsymbol{y}}_{E},
\end{aligned}
$$


where $\gamma$ is the flight path angle, $\chi$ is the course angle, and $\hat{\boldsymbol{x}}_{E}, \hat{\boldsymbol{y}}_{E}, \hat{\boldsymbol{z}}_{E}$ are unit vectors in the earth axes aligned with north, east, and down, respectively (see Fig. 1). In this kinematic coordinate system, an aircraft moving with true airspeed $V$ has the velocity $\boldsymbol{v}=V \hat{\boldsymbol{V}}$, from which one obtains the acceleration vector

$$
\dot{\boldsymbol{v}}=\dot{V} \hat{\boldsymbol{V}}+V \frac{d}{d t} \hat{\boldsymbol{V}}=\dot{V} \hat{\boldsymbol{V}}+V \dot{\gamma} \hat{\boldsymbol{\gamma}}+V \dot{\chi} \cos \gamma \hat{\boldsymbol{\chi}}
$$

With this expression, one can rather easily sum up the forces and obtain the equations of motion using Newton's second law.

In summary, the forces acting on the aircraft are gravity, thrust, and the aerodynamic forces of lift, drag, and side force. Further assuming that the force of gravity is aligned with $\hat{z}_{E}$, that thrust is entirely aligned with the aircraft body axis $\hat{\boldsymbol{x}}_{B}$, and following the standard convention for the directions of the lift, drag, and side forces, one obtains from Newton's second law the equations of motion:

$$
\begin{aligned}
\dot{V} & =\left(F_{T} \cos \alpha \cos \beta-F_{D}-\mathrm{m} g \sin \gamma\right) / \mathrm{m} \\
\dot{\gamma} & =\left(F_{\hat{a}_{L}} \cos \varphi-F_{\hat{a}_{Y}} \sin \varphi-\mathrm{m} g \cos \gamma\right) / \mathrm{m} V \\
\dot{\chi} & =\left(F_{\hat{a}_{L}} \sin \varphi+F_{\hat{\boldsymbol{a}}_{Y}} \cos \varphi\right) / \mathrm{m} V \cos \gamma .
\end{aligned}
$$

In these equations, $F_{T}$ is the net thrust produced by all engines, $F_{D}$ is the magnitude of the drag force, $\alpha$ is the angle of attack, $\beta$ is the sideslip angle, $g$ is acceleration due to gravity, and $\mathrm{m}$ is the mass of the aircraft. Finally,

$$
\begin{aligned}
F_{\hat{\boldsymbol{a}}_{L}} & =F_{T} \sin \alpha+F_{L} \\
F_{\hat{\boldsymbol{a}}_{Y}} & =F_{T} \cos \alpha \sin \beta+F_{Y},
\end{aligned}
$$

are the net force magnitudes aligned with the lift and side force direction vectors, while $F_{L}$ and $F_{Y}$ are the respective magnitudes of the lift and side force components. Fig. 1 illustrates the relationships between direction vectors and angles according to standard convention.
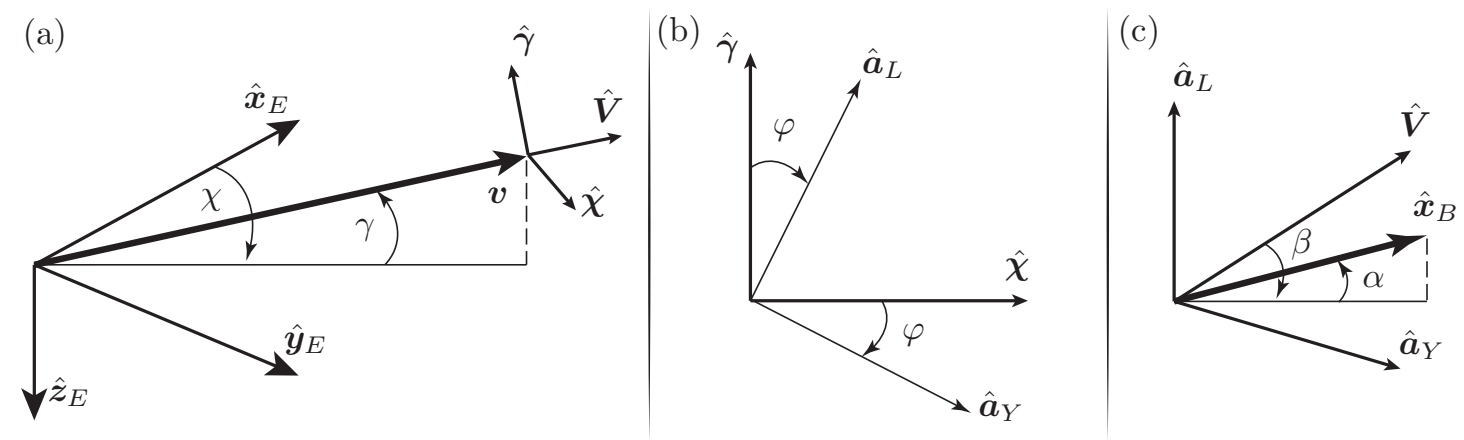

Figure 1: (a) 3D orientation of velocity vector $\boldsymbol{v}$ with respect to the earth fixed axes. (b) Roll angle $\varphi$ rotation to get from kinematic axes to aerodynamic axes, where $\hat{\boldsymbol{V}}$ is into the page. (c) Side-slip angle $\beta$ and angle of attack $\alpha$ rotations to go from the velocity axis $\hat{\boldsymbol{V}}$ to the body axis $\hat{\boldsymbol{x}}_{B}$.

\section{II.A. Maneuverability Model}

Next, parameterized expressions for the aerodynamic forces $F_{D}, F_{L}$, and $F_{Y}$ are developed. In general such expressions would depend on many parameters $(\alpha, \beta$, mass, air density, wind speed, etc.). However, one can obtain a simplified, but perhaps more useful model, by focusing on the primary dependencies through the 
model defined with

$$
\begin{aligned}
& F_{D}(\alpha)=\bar{q} \mathrm{~S} C_{D}(\alpha)=\bar{q} \mathrm{~S}\left(D_{0}+D_{1} \alpha+D_{2} \alpha^{2}\right) \\
& F_{L}(\alpha)=\bar{q} \mathrm{~S} C_{L}(\alpha)=\bar{q} \mathrm{~S}\left(L_{0}+L_{1} \alpha\right) \\
& F_{Y}(\beta)=\bar{q} \mathrm{~S} C_{Y}(\alpha)=\bar{q} \mathrm{~S}\left(Y_{0}+Y_{1} \beta\right),
\end{aligned}
$$

where $\bar{q}$ is dynamic pressure $\left(\bar{q}=\rho V^{2} / 2\right.$, where $\rho$ is air density), $\mathrm{S}$ is the net wing surface area, and $\left\{D_{k}\right\}_{k=0}^{2}$ and $\left\{L_{k}, Y_{k}\right\}_{k=0}^{1}$ are the expansion constants for the non-dimensional aerodynamic force coefficients $C_{D}, C_{L}$, and $C_{Y}$. Also note, for an undamaged aircraft one usually sets $Y_{0}=0$. Next inserting (5)-(7) into (2)-(4), and relying on the small angle approximations $\sin \beta \approx 0, \sin \alpha \cos \beta \approx 0$, and $\cos \alpha \cos \beta \approx 1$, one obtains the following coupled pair of nonlinear differential equations for the state vector $x=[V, \gamma]^{T}$

$$
f(x, u ; c)=\left[\begin{array}{c}
\dot{V} \\
\dot{\gamma}
\end{array}\right]=\left[\begin{array}{c}
\dot{x}_{1} \\
\dot{x}_{2}
\end{array}\right]=\left[\begin{array}{l}
-c_{1} \kappa x_{1}^{2}-c_{2} \kappa u_{2} x_{1}^{2}-c_{3} \kappa u_{2}^{2} x_{1}^{2}+u_{1} / \mathrm{m}-g \sin x_{2} \\
c_{4} \kappa x_{1} \cos u_{3}+c_{5} \kappa u_{2} x_{1} \cos u_{3}-c_{6} \kappa u_{4} x_{1} \sin u_{3}-g \cos x_{2} / x_{1}
\end{array}\right],
$$

where $u=\left[F_{T}, \alpha, \varphi, \beta\right]^{T}$ is the virtual input vector,

$$
c=\left[D_{0}, D_{1}, D_{2}, L_{0}, L_{1}, Y_{1}\right]^{T}
$$

is the aerodynamic parameter vector, and where $\kappa=\mathrm{S} \rho / 2 \mathrm{~m}$. Furthermore, the remaining possibly time varying parameters $(g, \mathrm{~m}, \mathrm{~S}, \rho)$ are assumed effectively know from measurement or separate estimation process. ${ }^{\mathrm{a}}$ We refer to such a model as a maneuverability model because of its dependence on virtual inputs rather than direct control inputs, which if needed can be determined by leveraging the principle of time-scale separation. ${ }^{11}$ The virtual inputs are however the parameters most closely related to any overall determination of maneuverability for the slower aircraft dynamics covering the motion of the center of mass. Furthermore, this model has a variety of properties extremely useful to applications, which we will bring to light in much of the discussion to follow.

\section{II.B. Trim}

The set of trimmable aircraft states is defined by $\{x \mid f(x, u ; c)=0,(x, u) \in \mathcal{B}\}$, where $\mathcal{B}$ represents the set of overall allowable states and virtual inputs. In addition, $\mathcal{B}$ should limit $(x, u)$ to the domain over which the model is valid. A common approach for discovering trimmable states is to solve some variation of

$$
u=\underset{u \in \mathcal{B}}{\operatorname{argmin}}\|f(x, u ; c)\|,
$$

for any desired fixed $x$, using standard constrained optimization routines. ${ }^{22,23}$ While this approach can work for almost arbitrarily sophisticated aircraft models, its application to map the entire trim envelope is numerically intensive, and requires special care when the local minima is not equal to zero. However, in stark contrast to this local optimization based approach, the specific maneuverability model (8) enables a far better analytical approach, capable of mapping the $(V, \gamma)$-trim envelope in tens of milliseconds ${ }^{\mathrm{b}}$ for any fixed aerodynamic coefficient vector $c$. This is an important capability because the set of trimmable states represents an a-priori safe aircraft maneuverability envelope.

Characterizing the set of trimmable points for fixed $c$, then, requires setting the top and bottom equations on the right hand side of (8) equal to zero. The bottom equation is solved for $u_{2}$ (angle of attack) in terms of the other variables in that equation, which do not include $u_{1}$ (thrust). The top equation is then solved for $u_{1}$ and we substitute the previous solution for $u_{2}$. The result is a closed form expression for the required thrust and angle of attack needed to achieve trim for any given state and remaining virtual inputs. This enables a fast numerical sweep to determine the typically non-convex trim envelope as follows:

\footnotetext{
${ }^{\text {a }}$ In our implementation, we consider $(g, \mathrm{~m}, \mathrm{~S}, \rho)$ as part of the virtual input vector $u$.

${ }^{\mathrm{b}}$ Using current commercially available laptop computational capability.
} 
1. Setup a grid of state values in $\mathcal{B}$. For most practical applications a coarse resolution is sufficient.

2. Fix values for the roll angle $\varphi=u_{3}$, and the side-slip angle $\beta=u_{4}$.

3. For each point in the state grid, solve for the thrust $u_{1}$ and angle of attack $u_{2}$ needed to achieve trim.

4. Return only those trim points for which $F_{T}$ and $\alpha$ are within $\mathcal{B}$.

A crude visualization of the $(V, \gamma)$-trim envelope is then obtained by simply plotting the trimmable points from the above calculation. In addition, one should check whether the achieved trim points are stable. This requires checking the eigenvalues associated with the local linear approximation to (8) at each trim point [24, Thm. $2.1 \mathrm{pg}$. 36] and as before a closed form expression can be found, but it is too unwieldy to include here. The entire computation is fast enough to enable dynamic re-computation of the trim envelope as aircraft conditions change, or to compute extended envelopes by sweeping over values for $\varphi$ and $\beta$.

As an example, we set up a scenario using the Research Civil Aircraft Model (RCAM) ${ }^{25}$ in landing configuration (flaps and gear down, air density at sea level, etc.). Table 1 shows the parameter values used for the setup. The actual trim envelope calculation for a high resolution grid covering 409149 points was completed in $114 \mathrm{~ms}$ on a $2.6 \mathrm{GHz}$ MacBook Pro. The calculation included a check for stability, and in this case, all of the trimmable points were stable (but in general this may not be true). The calculated envelope is shown in Fig. 2.

Table 1: Parameter values for an example maneuverability envelope calculation.

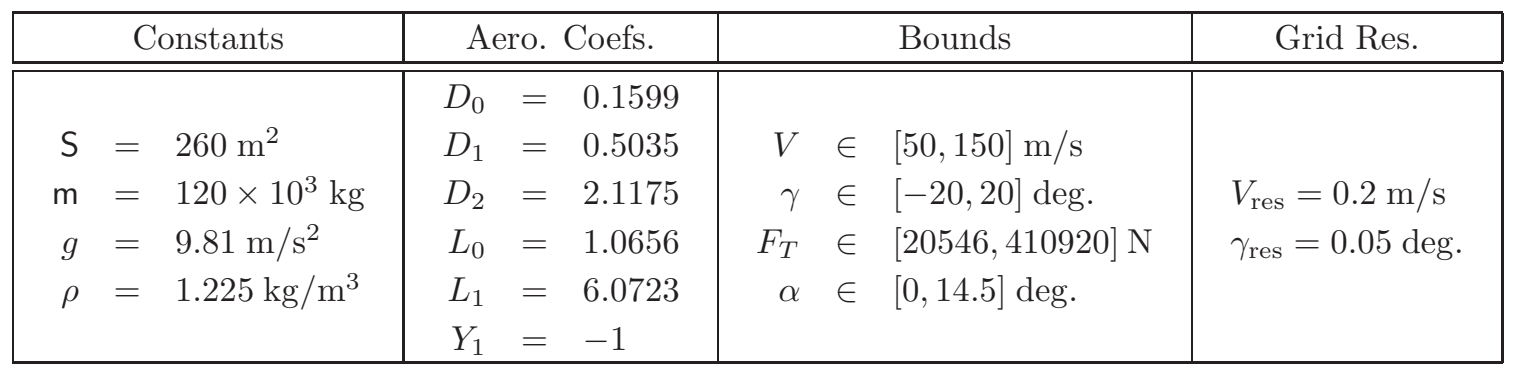
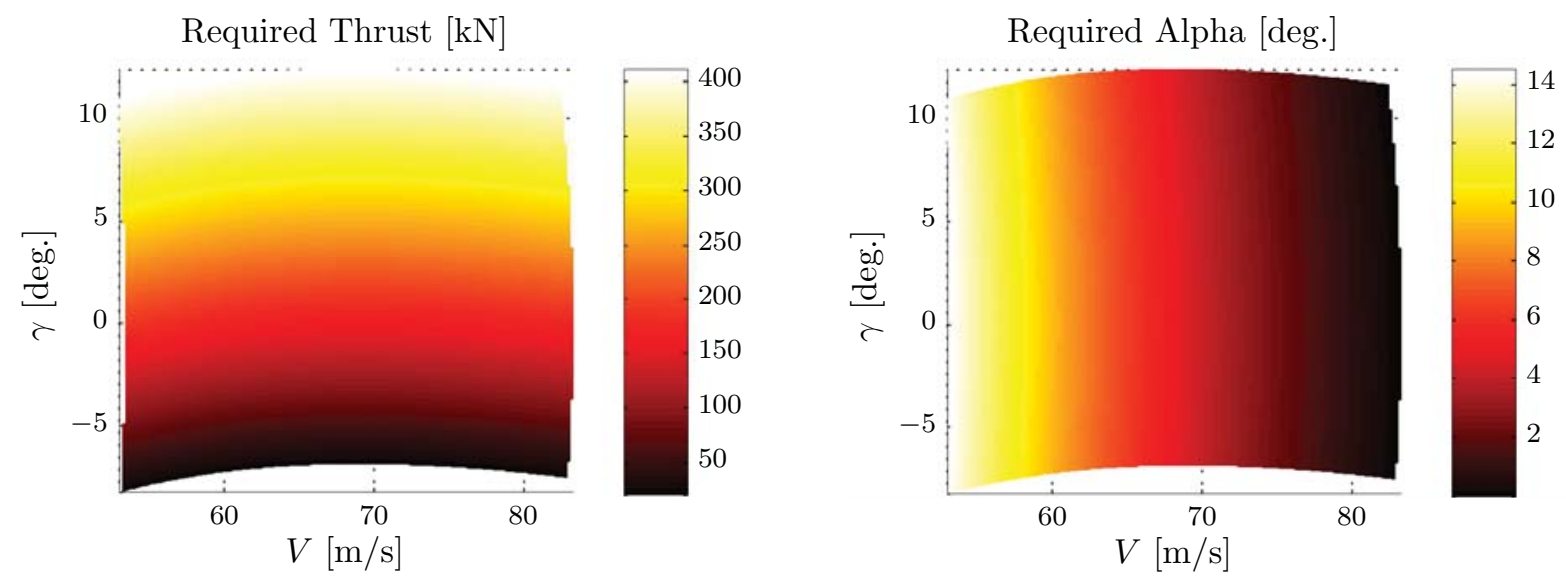

Figure 2: Maneuverability trim envelope for a representative commercial aircraft (similar to a B767 or A300) in landing configuration with $\varphi=\beta=0$. The plot on the left shows the maneuverability shaded according to the thrust required for trim, while the plot on the right shows the same envelope shaded according to required $\alpha$. 
In Fig. 2, it can be seen that the trim envelope boundaries are imposed by the input saturation limits. The upper boundary corresponds to maximum thrust $F_{T_{\max }}=410920 \mathrm{~N}$, and the lower boundary is imposed by minimum thrust $F_{T_{\min }}=20546 \mathrm{~N}$. The range for the angle of attack causes the left $\left(\alpha_{\max }=14.5^{\circ}\right.$, prior to stall) and right $\left(\alpha_{\min }=0^{\circ}\right)$ boundaries. It can also be observed that more thrust is needed for larger flight path angles, since the thrust force has to counteract the aircraft weight, and that a larger angle of attack occurs for slower speeds. Analyzing the range of thrust values over airspeed for constant flight path angle, shows that more thrust is needed for a further speed decrease below $69 \mathrm{~m} / \mathrm{s}$. This region corresponds to the range of the angle of attack $\alpha>4.5^{\circ}$. Analysis of the Lift-Drag Polar and the power required curve, shown in Fig. 3, confirms that this region is the back side of the power curve, and that $V=69 \mathrm{~m} / \mathrm{s}$ is the minimum drag airspeed.

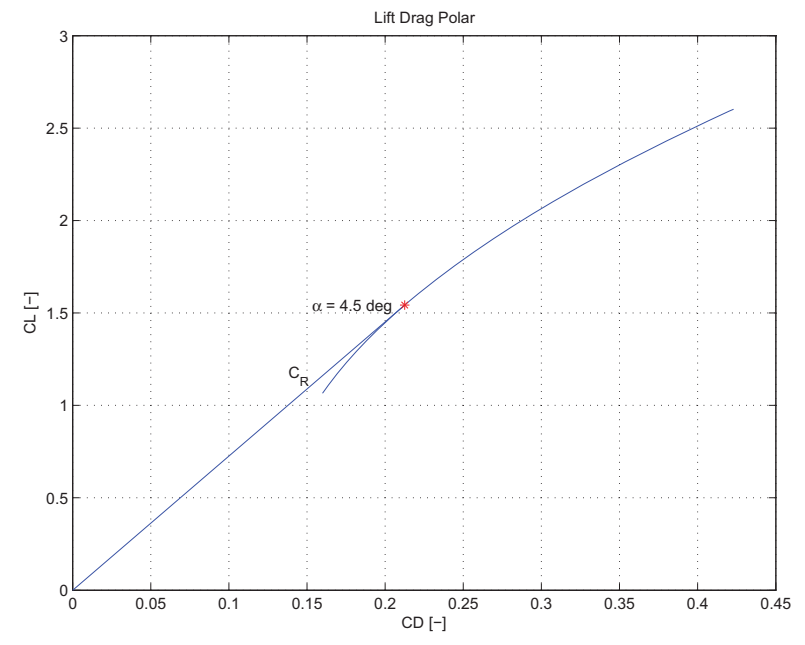

(a) Lift-Drag Polar
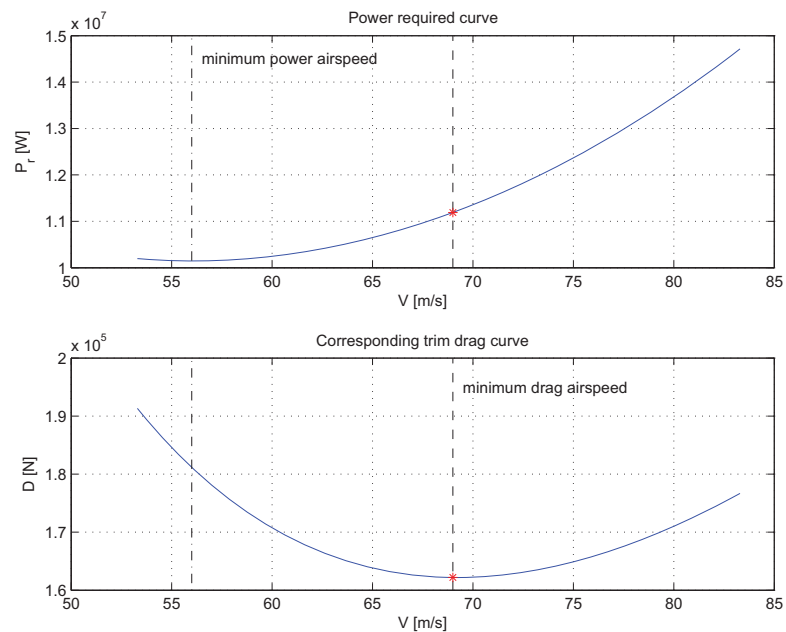

(b) Power required curve

Figure 3: The Lift-Drag polar and power requirement curves of the RCAM model confirm that the minimum drag airspeed is $V=69 \mathrm{~m} / \mathrm{s}$, and the region to the left is the back side of the power curve.

\section{II.C. Damage Scenarios}

Damage can be injected into our maneuverability model through two primary mechanisms. First, damage leading to diminished control authority is modeled through a constriction of the set $\mathcal{B}$ of overall allowable states and virtual inputs. For example, the maximum permissible angle of attack may diminish due to icing, or the maximum available thrust may be reduced due to engine damage. The second mechanism is through changes to the aerodynamic parameter vector $c$, and this will likely occur in conjunction with the first mechanism. This occurrence may for example model a decrease in the conversion between $\alpha$ and the generation of lift with perhaps an additional cost in drag.

To illustrate, Fig. 4 shows the effect of reduced aerodynamic capability, modeled by a $20 \%$ decrease to lift and $20 \%$ increase to drag, along with a $50 \%$ reduction to available thrust. For these damage scenarios, it can be seen in the left plot of Fig. 4 that a $20 \%$ decrease in lift combined with a $20 \%$ increase in drag results in a shift of the trim envelope towards higher airspeeds and lower flight path angles. This is physically explained by the force equilibrium equations:

$$
\begin{aligned}
C_{L}(\alpha) \rho V^{2} \mathrm{~S} / 2 & =W \cos \gamma \approx W \\
F_{T}-C_{D}(\alpha) \rho V^{2} \mathrm{~S} / 2 & =W \sin \gamma \approx W \gamma
\end{aligned}
$$

Due to the reduced lift capability, (10) shows that a higher airspeed is needed to compensate for the aircraft weight $W=\mathrm{m} g$. On the other hand, an increase in drag means that reduced net excess thrust is available 

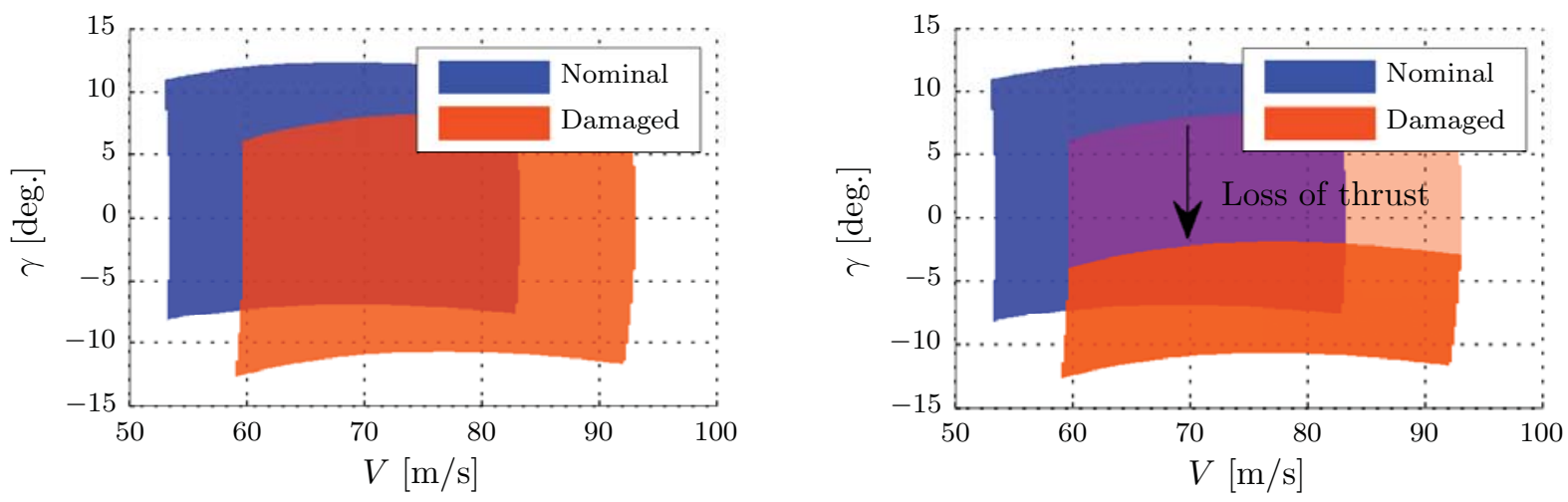

Figure 4: Trim envelope calculation for nominal and damaged cases with $\varphi=\beta=0$. The damaged case on the left corresponds to a $20 \%$ decrease of all the lift coefficients $\left\{L_{k}\right\}$, and a $20 \%$ increase of the drag coefficients $\left\{D_{k}\right\}$. The plot on the right shows the same damage case with an additional $50 \%$ loss of available thrust.

for climb in (11). If the maximum thrust limit $F_{T_{\max }}$ is reduced by $50 \%$, then there is a further reduction in net excess thrust available for climb, which is translated into a shift downwards of the upper limit of the trim envelope, as shown on the plot to the right in Fig. 4. Furthermore, this $50 \%$ reduction to $F_{T_{\max }}$ makes establishing trimmed level flight impossible in this scenario.

The maneuverability model we just developed may not accurately capture the aerodynamics for many other damage scenarios. In this instance, alternate parameterizations of the aerodynamic force equations (5)-(7) may be required, and existing research is in place to support this task. ${ }^{26-28}$ These extended cases can be treated by a system identification procedure capable of using observed flight data to select between competing damage models, like the one developed in the next section.

\section{System Identification}

The maneuverability model (8) is characterized by a vector of aerodynamic parameters $c$, which once known, ultimately determines the trim envelope as discussed above. A critical feature of the same maneuverability model, is that it is linear in these parameters for given state and virtual inputs. Therefore, the application of almost any system identification algorithm, say from [29], should yield quite satisfactory results with sufficient input excitation to cover the full dynamic response of the aircraft. The question of input or maneuver design for system identification is important and covered in the Refs. [26-31], along with additional methods for developing more sophisticated aerodynamics models and online parameter retrieval.

However, many important applications for online maneuverability characterization are aligned with tracking nominal performance, and identifying degradation to the envelope caused by impairment, during normal operations that would not constantly explore the space of possible inputs. Therefore, in an effort to enable progressive learning of the aircraft dynamics with the possibility of insufficient excitation, a novel Bayesian method was developed with the following high-level features:

- works in the background from pilot inputs and measurements collected while maneuvering naturally,

- leverages optimization and probability theory to simultaneously estimate both system parameters and uncertainty from the measured data,

- is biased towards greater uncertainty quantification, especially when excitation is insufficient,

- seamlessly enables selection between alternate impairment models through the evidence calculation. 
The objectives of this section, then, are to develop fast methods for estimating the aerodynamic coefficient vector $c$ from measured state and input data, to quantify the resulting estimation uncertainty, and to enable the comparison between competing models that explain the data.

\section{III.A. Measurement Model}

Our system identification algorithm stems from a midpoint-Euler discrete approximation for the measured state process:

$$
x(k+1)=x(k)+h f(\tilde{x}, u ; c)+\tau(k),
$$

where $h$ is the time-sample resolution, $\tilde{x}=[x(k)+x(k+1)] / 2$ and $\tau(k) \sim \mathcal{N}\left(0, h S^{-1}\right)$, for $k=0,1, \ldots, m-$ 1. While the maneuverability model is linear in $c$, it is still nonlinear in $x$ and $u$, and the midpointEuler approximation enables better representation of the true nonlinear system, for negligible additional computational cost. Furthermore, the more general case is developed where $x(k), \tau(k) \in \mathbb{R}^{n}$ and $c \in \mathbb{R}^{d}$; and this makes the method readily applicable to broader more sophisticated dynamics models. Clearly, $n=2$ and $d=6$ for the specific maneuverability model discussed above. Finally, the inverse covariance matrix $S$ is an important statistical parameter that characterizes the process noise $\tau(k)$, which we will also estimate from the observed data. This matrix is sometimes also referred to as the precision or information matrix, and we use these terms interchangeably in the discussion to follow.

\section{III.A.1. Sensor Measurement Error}

A more comprehensive approach would also model the effect of additive measurement noise to the states and inputs:

$$
\begin{aligned}
& y_{x}(k)=x(k)+\eta_{x}(k) \\
& y_{u}(k)=u(k)+\eta_{u}(k),
\end{aligned}
$$

where $\eta_{x, u}$ may be an independent additive Gaussian process. However, we will avoid this consideration by assuming the variance of $\tau(k)$ far exceeds that of $\eta_{x, u}$, which is not an impractical assumption in many cases. With additional sophistication, our approach is extendable to include the measurement noise as well.

\section{III.B. Probabilistic Approach}

Our objectives are met by using the measurement model (12) to develop an expression for the posterior probability density function (pdf)

$$
p(c, S \mid \mathbf{x}, \mathbf{u}, x(0)),
$$

where $\mathbf{x}$ is short-hand for $[x(1), x(2), \ldots, x(m)]$ and $\mathbf{u}$ is short-hand for $[u(0), u(1), \ldots, u(m-1)]$. This is the pdf that describes the joint set of reasonable aerodynamic parameters $c$ and process noise precision matrices $S$, given the available data $(\mathbf{x}, \mathbf{u}$, and $x(0))$. The remainder of this section outlines the basic derivation of the posterior pdf. Then, starting in §III.C, a fast algorithm to find the estimates for $c$ and $S$ that maximize this posterior distribution is presented, along with analytical approximations for quantifying the estimation uncertainty.

Using Bayes' Theorem the posterior distribution is decomposed as follows:

$$
p(c, S \mid \mathbf{x}, \mathbf{u}, x(0))=\frac{p(c, S, \mathbf{x} \mid \mathbf{u}, x(0))}{\mathcal{Z}}=\frac{p(\mathbf{x} \mid c, S, \mathbf{u}, x(0)) p(c, S \mid \mathbf{u}, x(0))}{\mathcal{Z}},
$$

where we refer to $p(\mathbf{x} \mid c, S, \mathbf{u}, x(0))$ as the likelihood distribution, $p(c, S \mid \mathbf{u}, x(0))$ as the prior distribution, and

$$
\mathcal{Z}=\int_{c^{\prime}, S^{\prime}} p(c, S, \mathbf{x} \mid \mathbf{u}, x(0)) d c^{\prime} d S^{\prime}
$$

as the evidence. The evidence $\mathcal{Z}=p(\mathbf{x} \mid \mathbf{u}, x(0))$ is the probability density of the observed state data given the input measurements, initial state, and all implied modeling assumptions. In essence, it is the probability of 
the observations given the model. This is an important quantity because the evidence for alternative models can be computed and compared; a process that would allow one to extend the present framework to consider alternate maneuverability models that may arise in damage scenarios, as previously discussed in II.C.

\section{III.B.1. Priors}

The prior distribution is assumed to take the following form

$$
p(c, S \mid \mathbf{u}, x(0))=p(c, S)=p(S) p(c) .
$$

This distribution characterizes the available information prior to making any measurements, and the equation implies prior independence of the parameters, inputs, and initial state.

The prior on the aerodynamic parameter vector $c$ is further assumed to follow a multivariate normal distribution,

$$
p(c)=(2 \pi)^{-d / 2}\left|\Sigma_{c}\right|^{-1 / 2} \exp \left\{-\frac{1}{2}\left(c-\mu_{c}\right)^{T} \Sigma_{c}^{-1}\left(c-\mu_{c}\right)\right\},
$$

where $\mu_{c}$ and $\Sigma_{c}$ are used to set the prior knowledge of the model parameters.

The prior on the precision matrix $S$ is assumed to follow a specialized version of the Wishart distribution:

$$
p(S)=\Psi_{n+1} \exp \left\{-\frac{1}{2} \operatorname{tr}\left(\Lambda^{-1} S\right)\right\}
$$

where $\Psi_{n+1}$ is the known normalizing constant of integration, and $\Lambda \in \mathbb{S}_{++}^{2}$ is a given matrix that enables an encoding of prior information as needed. ${ }^{c}$ Typically, one would set $\Lambda$ to a diagonal matrix related to a prior worst case assessment of expected model error and turbulence. This creates a pessimistic prior on $S$ that forces a justification of uncertainty through measurement. To see how this works, consider the case where the airspeed and flight path angle errors are independent and expected to be $\lambda_{1}$ and $\lambda_{2}$, respectively. Note, these are error variances based on how well one expects the model to perform before any new measurements are taken; and if this is unknown one should set $\lambda_{1}$ and $\lambda_{2}$ to cover the range of possible values (usually known from the underlying physics of the problem). Setting $\Lambda=\operatorname{diag}\left(\lambda_{1}, \lambda_{2}\right)$, the Wishart prior looks like,

$$
p(S) \propto \exp \left(-\frac{S_{11}}{2 \lambda_{1}}\right) \exp \left(-\frac{S_{22}}{2 \lambda_{2}}\right)
$$

where $S_{11}$ and $S_{22}$ are the diagonal elements of $S$. This, however, is equivalent to the case where $S_{11}$ and $S_{22}$ are each independently distributed according to an exponential distribution with means $2 \lambda_{1}$ and $2 \lambda_{2} \cdot{ }^{\mathrm{d}}$ Furthermore, exponential priors assign higher probability density to small values for $S_{11}$ or $S_{22}$, and since $S$ is an inverse covariance matrix this corresponds to larger variances in the expected model performance. This is what we mean by pessimistic prior, and it is a key feature of our approach.

The ability to leverage prior information in the probabilistic formulation enables a number of distinct advantages especially when used in combination with evidence calculations. For example, in a nominal aircraft one may have a high degree of confidence in the values of the aerodynamic parameters $c$ from flight and wind tunnel testing, which can be encoded into $p(c)$. In this case, the observed data will support the model with a sharp prior and the corresponding evidence calculation will be high. However, in the presence of damage the aerodynamic parameters will shift, contradict the prior, diminish the evidence calculation, and force the consideration of alternative models.

\footnotetext{
${ }^{\mathrm{c}}$ This corresponds to the general Wishart distribution with $n+1$ degrees of freedom.

${ }^{\mathrm{d}}$ In this case, there is also no prior preference on $S_{12}$ other than the implicit constraint required for $S$ to be positive definite (i.e., $S_{11} S_{22}-S_{12}^{2}>0 \Longrightarrow-\sqrt{S_{11} S_{22}}<S_{21}<\sqrt{S_{11} S_{22}}$ ).
} 


\section{III.B.2. State Likelihood}

Next we develop the likelihood function for the states given the aerodynamic parameters, precision matrix, virtual inputs, and initial state. Following Ref. [32], this is accomplished by first noting the system of equations induced by (12), represents a nonlinear transformation of random variables, from $\tau(k)$ to $x(k+1)$ for $k=0, \ldots, m-1$. The corrected likelihood pdf is thus given by:

$$
p(\mathbf{x} \mid c, S, \mathbf{u}, x(0))=|J| \prod_{k=0}^{m-1}(2 \pi h)^{-n / 2}|S|^{1 / 2} \exp \left\{-\frac{1}{2 h} b(k)^{T} S b(k)\right\},
$$

where,

$$
b(k)=x(k+1)-x(k)-h f(\tilde{x}(k), u(k) ; c),
$$

and where $J$ is the $m n \times m n$ Jacobian matrix accounting for the full transformation. For small $h$, the determinant of $J$ is well approximated by

$$
|J| \approx \exp \left\{-\frac{h}{2} \sum_{j=0}^{m-1} \operatorname{tr} F(\tilde{x}(j), u(j) ; c)\right\},
$$

where $F(x, u ; c)$ is the $n \times n$ Jacobian matrix of the vector field $f$ with respect to $x$. One should notice that $\operatorname{tr} F=\nabla \cdot f$, which is the divergence of the vector field defined by $f$.

Many nonlinear dynamical models, including the maneuverability model (8), can be expressed as affine functions of the unknown model parameters $[32, \S I I . D]^{\mathrm{e}}$, generally taking the form

$$
f(x, u ; c)=U(x, u) c+v(x, u)
$$

and when this is the case one can also write,

$$
\operatorname{tr} F(x, u ; c)=q(x, u)^{T} c+r(x, u) .
$$

Thus, the nonlinear dynamics are characterized as a combination of the columns of $U(x, u)$, with unknown weights $c$, along with a known part specified by $v(x, u)$.

\section{III.C. Estimation Algorithm}

With all the required distributions now defined we have two remaining tasks. The first is to find the maximum a posteriori (MAP) estimates for the aerodynamic parameter vector $c$ and precision matrix $S$ by solving

$$
\text { maximize } \ln p(c, S \mid \mathbf{x}, \mathbf{u}, x(0))=\ln p(\mathbf{x} \mid c, S, \mathbf{u}, x(0))+\ln p(c)+\ln p(S)-\ln \mathcal{Z},
$$

where $\ln \mathcal{Z}$ is a constant that can be ignored for this step. The second task is to determine an approximation for the marginal posterior distribution $p(c \mid \mathbf{x}, \mathbf{u}, x(0))$.

Finding the MAP estimate is equivalent to minimizing the negative log of the joint $\operatorname{pdf} p(c, S, \mathbf{x} \mid \mathbf{u}, x(0))$ with $\mathrm{x}$ fixed by solving:

$$
\text { minimize } f_{0}(c, S)=-\ln p(c, S, \mathbf{x} \mid \mathbf{u}, x(0))
$$

with respect to the variables $c \in \mathbb{R}^{d}$ and $S \in \mathbb{S}_{++}^{n}$ and where,

$$
\begin{aligned}
f_{0}(c, S)=\frac{m n}{2} & \ln 2 \pi h-\frac{m}{2} \ln |S|+\frac{h}{2} \sum_{k=0}^{m-1} q_{k}^{T} c+r_{k}+\left(U_{k} c+v_{k}\right)^{T} S\left(U_{k} c+v_{k}\right) \\
+ & \frac{1}{2} \ln \left|(2 \pi)^{d} \Sigma_{c}\right|+\frac{1}{2}\left(c-\mu_{c}\right)^{T} \Sigma_{c}^{-1}\left(c-\mu_{c}\right)+\frac{1}{2} \operatorname{tr}\left(\Lambda^{-1} S\right)-\ln \Psi_{n+1} .
\end{aligned}
$$

In this expression, $q_{k}=q(\tilde{x}(k), u(k)), r_{k}=r(\tilde{x}(k), u(k)), U_{k}=U(\tilde{x}(k), u(k)), v_{k}=v(\tilde{x}(k), u(k))-\Delta x(k)$, and $\Delta x(k)=[x(k+1)-x(k)] / h$. One way to accomplish the minimization (24) is by using the following block coordinate descent algorithm:

\footnotetext{
${ }^{\mathrm{e}}$ If an exact representation is not possible, then usually the nonlinear model can at least be approximated in this manner.
} 
1. Start from an initial guess $\left(c^{0}, S^{0}\right)$, and $k=0$.

2. Precision Update. $S^{k+1}=\operatorname{argmin}_{S \in \mathbb{S}_{++}^{n}} f_{0}\left(c^{k}, S\right)$.

3. Parameter Update. $c^{k+1}=\operatorname{argmin}_{c} f_{0}\left(c, S^{k+1}\right)$.

4. Repeat until $\Delta c^{T} M \Delta c<\epsilon$, where $\Delta c=c^{k+1}-c^{k}$, and $M$ is defined by (26) below.

It can be shown that each update step requires solving a well-posed convex optimization problem and this condition is sufficient to guarantee convergence of the overall algorithm to a joint local stationary solution [33, §2.7, pg. 272]. Furthermore, by separately setting the gradient with respect to $S$ and $c$ equal to zero, one finds, respectively, that the precision update has a closed form analytical expression, and the parameter update requires solving a well posed regularized least-squares problem. While we can not guarantee global joint optimality in both $c$ and $S$, the algorithm does uniquely determine the global optimal precision matrix corresponding to any fixed estimate for $c$ (and vice-versa), and in practice the algorithm has demonstrated rapid convergence to reliable estimates.

In keeping with the probabilistic approach, one may desire to stop the algorithm when $\Delta c$ is insignificant with respect to the uncertainty in the current parameter estimate $c^{k}$. This prevents needlessly updating the current estimate when the uncertainty is large compared to the update magnitude. One can show that

$$
M=\left(\Sigma_{c}^{-1}+h \sum_{k=0}^{m-1} U_{k}^{T} S^{k+1} U_{k}\right)
$$

specifies the approximate multivariate precision matrix for the current parameter estimate. Therefore, the stopping criterion that $\Delta c^{T} M \Delta c<\epsilon$, ensures the update falls within a small uncertainty ellipsoid around the current parameter estimate. In fact, one can show that $\operatorname{Prob}\left\{\Delta c^{T} M \Delta c<\epsilon\right\}=F_{\chi_{d}^{2}}(\epsilon)$, where $F_{\chi_{d}^{2}}$ is the cumulative distribution function (cdf) of a chi-squared distribution with $d$ degrees of freedom. In particular, we used $\epsilon=0.001$, for which $F_{\chi_{d}^{2}}(\epsilon) \approx 2 \times 10^{-11}$, in our examples. ${ }^{\mathrm{f}}$

Finally, an important consequence of the convexity inherent to both the precision and parameter updates, is that a variety of hard constraints can be incorporated into the problem. In particular, if the constraints are also convex then the overall convergence properties of the algorithm are unaffected (see Refs. [33] and [34] for further details), although in some cases more careful analysis of the estimation uncertainty may be required. For example, known trim points are easily handled by introducing linear equality constraints into the parameter update step.

\section{III.D. Parameter Uncertainty}

Determining the estimation uncertainty amounts to finding a quadratic approximation for the negative log of the joint distribution and normalizing appropriately. This is readily accomplished by forming a quadratic second order taylor approximation around the optimal estimate. For (25), the general form is

$$
\tilde{f}_{0}(c, s)=f_{0}\left(c^{\star}, S^{\star}\right)+\frac{1}{2}\left[\begin{array}{c}
c-c^{\star} \\
s-s^{\star}
\end{array}\right]^{T}\left[\begin{array}{cc}
\nabla_{c}^{2} f_{0} & \nabla_{c s} f_{0} \\
\nabla_{c s} f_{0}^{T} & \nabla_{s}^{2} f_{0}
\end{array}\right]\left[\begin{array}{c}
c-c^{\star} \\
s-s^{\star}
\end{array}\right],
$$

where the gradient term is omitted because it is approximately zero at the MAP parameter estimate $\left(c^{\star}, s^{\star}\right)$. Furthermore, $S$ is expressed through the expansion

$$
S=\sum_{j=1}^{z} s_{j} E_{j}
$$

\footnotetext{
${ }^{\mathrm{f}}$ To find the value of $\epsilon$ that produces, say, a $0.1 \%$ confidence ellipsoid we simply lookup or numerically compute $\epsilon=$ $F_{\chi_{d}^{2}}^{-1}(0.001)$. For example, this is accomplished in MATLAB ${ }^{\circledR}$ using chi2inv $(0.001$, d).
} 
where the $E_{j} \in \mathbb{S}^{n}$ serve as basis matrices for the space of possible precision matrices. ${ }^{\mathrm{g}}$ After substituting, (28) into (25), the expressions for $\nabla_{c}^{2} f_{0}, \nabla_{c s} f_{0}$, and $\nabla_{s}^{2} f_{0}$ can be found. In particular, one may readily observe that $\nabla_{c}^{2} f_{0}=M$, where $M$ is defined by (26) evaluated with $S^{k+1}=S^{\star}$.

With these expressions, we find from (27) that the approximate full posterior distribution is

$$
\tilde{p}(w \mid \mathbf{x}, \mathbf{u}, x(0))=\left|(2 \pi)^{d+z} \Sigma\right|^{-1 / 2} \exp \left\{-\frac{1}{2} w^{T} \Sigma^{-1} w\right\},
$$

where $w=\left(c-c^{\star}, s-s^{\star}\right) \in \mathbb{R}^{d+z}$, and with $H=-\nabla_{s}^{2} \ln |S|$,

$$
\Sigma^{-1}=\left[\begin{array}{cc}
\nabla_{c}^{2} f_{0} & \nabla_{c s} f_{0} \\
\nabla_{c s} f_{0}^{T} & \frac{m}{2} H
\end{array}\right] .
$$

The approximate marginal posterior distribution for the aerodynamic parameter vector is then,

$$
\tilde{p}(c \mid \mathbf{x}, \mathbf{u}, x(0))=\left|(2 \pi)^{d} \Sigma^{\prime}\right|^{-1 / 2} \exp \left\{-\frac{1}{2}\left(c-c^{\star}\right)^{T} \Sigma^{\prime-1}\left(c-c^{\star}\right)\right\},
$$

where $\Sigma^{\prime-1}=\nabla_{c}^{2} f_{0}-(2 / m) \nabla_{c s} f_{0} H^{-1} \nabla_{c s} f_{0}^{T}$. Asymptotically, as $m \rightarrow \infty$, we see $\Sigma^{\prime-1} \approx \nabla_{c}^{2} f_{0}=M$, and observe in (26) that $M$ is essentially the prior information matrix updated by the data information matrix. Also, for large $m$ we have that $\tilde{p}(c \mid \mathbf{x}, \mathbf{u}, x(0)) \approx \tilde{p}\left(c \mid S^{\star}, \mathbf{x}, \mathbf{u}, x(0)\right)$. That is, the marginal posterior for the aerodynamic coefficient vector is approximately the conditional distribution evaluated at the precision matrix estimate. Furthermore, $\tilde{p}\left(c \mid S^{\star}, \mathbf{x}, \mathbf{u}, x(0)\right)=p\left(c \mid S^{\star}, \mathbf{x}, \mathbf{u}, x(0)\right)$ exactly, because $f_{0}$ is quadratic in $c$.

\section{III.E. Evidence}

The evidence calculation is derived from (27) as follows. The approximation for the joint pdf is

$$
\begin{aligned}
p(c, S, \mathbf{x} \mid \mathbf{u}, x(0)) & =\exp \left\{-f_{0}(c, S)\right\} \\
& \approx \exp \left\{-\tilde{f}_{0}(c, s)\right\} \\
& =\exp \left\{-f_{0}\left(c^{\star}, S^{\star}\right)\right\} \exp \left\{-\frac{1}{2} w^{T} \Sigma^{-1} w\right\} \\
& =p\left(c^{\star}, S^{\star}, \mathbf{x} \mid \mathbf{u}, x(0)\right) \exp \left\{-\frac{1}{2} w^{T} \Sigma^{-1} w\right\} .
\end{aligned}
$$

The evidence is then obtained by integrating the approximate expression over all possible values for $w=(c, s)$. The result in log-form is

$$
\begin{aligned}
\ln Z & \approx \ln p\left(c^{\star}, S^{\star}, \mathbf{x} \mid \mathbf{u}, x(0)\right)+\frac{1}{2} \ln \left|(2 \pi)^{d+z} \Sigma\right| \\
& =-f_{0}\left(c^{\star}, S^{\star}\right)+\frac{1}{2} \ln \left|(2 \pi)^{d+z} \Sigma\right|,
\end{aligned}
$$

where $f_{0}$ is given by (25). The accuracy of this evidence estimate can be checked using non-deterministic methods such as importance sampling [35, §13.3].

\section{III.F. Extensions}

The system identification method developed so far is amenable to a variety of extensions. An important example is the incorporation of direct accelerometer measurements. This can be accomplished, for example, by introducing an accelerometer measurement process model defined by,

$$
a(k)=A_{k} c+\tau_{a},
$$

${ }^{g}$ While all of $\mathbb{S}^{n}$ is covered by $z=n(n+1) / 2$ such basis matrices, there are implicit (convex) constraints on the feasible values $s \in \mathbb{R}^{z}$ can take, since $S$ must also be positive definite. 
where $a(k)=\left[F_{D}, F_{L}, F_{Y}\right]^{T} / \mathrm{m}$ represents the component aerodynamic accelerations (corrected for gravity and thrust) observed at each time instance $k, A_{k}$ is the corresponding matrix from (5)-(7) defined as

$$
A_{k}=\kappa x_{1}(k)^{2}\left[\begin{array}{cccccc}
1 & \alpha(k) & \alpha(k)^{2} & 0 & 0 & 0 \\
0 & 0 & 0 & 1 & \alpha(k) & 0 \\
0 & 0 & 0 & 0 & 0 & \beta(k)
\end{array}\right]
$$

and $\tau_{a}$ is the associated measurement noise vector distributed $\mathcal{N}\left(0, S_{a}^{-1}\right)$. Here, $S_{a}$ is a separate unknown precision matrix for the accelerometer error. Recall from before that $x_{1}(k)$ is the total airspeed (which is the first element of the state vector), and $\kappa=\mathrm{S} \rho / 2 \mathrm{~m}$. Now, the full joint distribution can be factored as

$$
\begin{aligned}
p\left(c, S, S_{a}, \mathbf{a}, \mathbf{x} \mid \mathbf{u}, x(0)\right) & =p\left(\mathbf{a}, \mathbf{x} \mid c, S, S_{a}, \mathbf{u}, x(0)\right) p\left(c, S, S_{a} \mid \mathbf{u}, x(0)\right) \\
& =p\left(\mathbf{a} \mid c, S_{a}, \mathbf{x}, \mathbf{u}, x(0)\right) p(\mathbf{x} \mid c, S, \mathbf{u}, x(0)) p(c) p(S) p\left(S_{a}\right),
\end{aligned}
$$

where $\mathbf{a}$ represents the full set of accelerometer measurements, analogous to $\mathbf{x}$ and $\mathbf{u}$. The second line in this factorization follows by applying the definition of conditional probability to factor $p(\mathbf{a}, \mathbf{x} \mid \cdot)$, and because the prior knowledge regarding $c, S$, and $S_{a}$ is assumed to be assembled from independent sources before accounting for any new measurements. A similar approach to the one outlined in §III.B.1-III.E can be followed to derive the estimation algorithm, this time with an additional Wishart prior characterized by $\Lambda_{a}$ (compare (18)) and update step for $S_{a}$, along with the calculations for uncertainty and evidence. ${ }^{\text {h }}$

Finally, we wish to emphasize the overall formalism developed here is readily applied to dynamical systems that are nonlinear in the state and input variables, but affine in the unknown system identification parameters. This characteristic leads to the analytical uncertainty and evidence approximations just discussed, which in turn make it possible to quickly evaluate competing models using standard techniques from Bayesian model selection [36, Ch. 4]. We will highlight one such example in the discussion to follow.

\section{III.G. Example}

The overall system identification approach just discussed is now demonstrated using an example that focuses on verifying our estimation algorithm and uncertainty quantification under the condition that all of our modeling assumptions hold. To accomplish this, we started by simulating the full nonlinear dynamics for a general transport class aircraft, similar to a B757, operating nominally at an altitude of 3048 meters for $90 \mathrm{~s}$. This was done only to obtain realistic values for the virtual inputs. Next, using these inputs, we generated state measurements exactly from our model (8) in time step increments of $0.01 \mathrm{~s}$, while also adding independent zero-mean Gaussian state noise with standard deviations of $3 \mathrm{~m} / \mathrm{s}$ and $0.5^{\circ}$ for $V$ and $\gamma$, respectively. Accelerometer measurements were also generated concurrently with the state measurements according to the model (32), with accelerometer measurement covariance $S_{a}^{-1}=0.1^{2} I$. The prior distribution on the parameter vector was set using

$$
\mu_{c}=[0,0.5,0,0,4.5,0]^{T} \quad \Sigma_{c}=\operatorname{diag}\left(2^{2}, 3^{2}, 3^{2}, 3^{2}, 3^{2}, 2^{2}\right),
$$

where $\operatorname{diag}(\cdot)$ denotes a diagonal matrix with diagonal elements defined by the input list. Also, the Wishart priors for $S$ and $S_{a}$ were characterized by diagonal matrices $\Lambda$ and $\Lambda_{a}$, based on assuming worst case standard deviations of $10 \mathrm{~m} / \mathrm{s}$ for $V, 5^{\circ}$ for $\gamma$, and $1 \mathrm{~m} / \mathrm{s}^{2}$ for the accelerometer measurements. Finally, to simulate a rapid fault onset, the first $45 \mathrm{~s}$ of our simulation corresponded to the nominal model with true underlying coefficients from Table 1, while the final $45 \mathrm{~s}$ were produced using coefficients from the $20 \%$ damage model discussed in §II.C. Fig. 5 shows the angular virtual inputs, state, and accelerometer measurements corresponding to our simulation.

Our estimation procedure was then applied to the above simulated data after decimation by a factor of 10 , so that the measurements correspond to a 0.1 second time sampling resolution. This decimation step was

\footnotetext{
${ }^{\mathrm{h}}$ The particular simplistic approach suggested here assumes independent state and accelerometer measurements. This can be realized, for example, by using GPS data to derive the state measurements separately from the accelerometer measurements.
} 

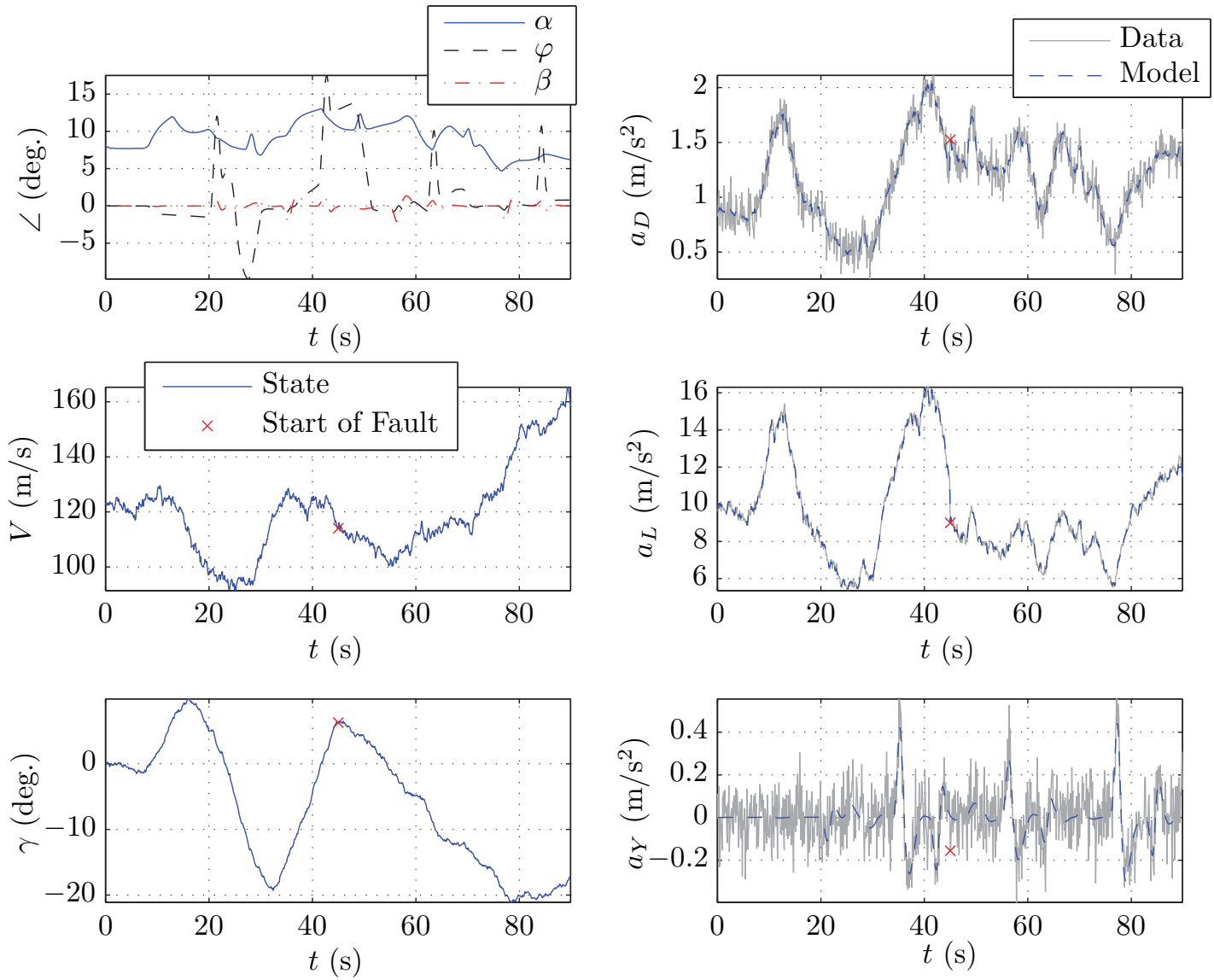

Figure 5: Left Column: virtual angular and state inputs to the estimation procedure produced using a general transport aircraft simulator. Right Column: measured accelerometer data and model fit at the estimated parameter values. The onset of a fault occurs $45 \mathrm{~s}$ into the simulation. The fault corresponds to $20 \%$ increase in all drag coefficients and a $20 \%$ decrease in all lift coefficients from the nominal case.

intended to test the feature of our estimation procedure that enables it to operate on time samples from a continuous nonlinear model. Fig. 6 shows the estimation results for the linear lift and drag coefficients after applying the algorithm separately to the nominal and damage cases. The same broad prior distribution was used for each case, and the algorithm processed each data set of 450 samples in about $100 \mathrm{~ms}$ converging in only 4 iterations of the main loop. These results demonstrate the algorithm's ability to recover the actual coefficients and quantify uncertainty. In this case, the damage is significantly more distinguishable in the linear lift coefficient than in the drag coefficient. Note also that the algorithm adaptively determines estimation uncertainty based on the data it receives, and in this example the measured data enables a more accurate quantification of the damage model coefficients.

So far our analysis has only examined the ability to discern the fault after knowing when it occurred. To demonstrate ability to identify the fault we turn to our evidence calculation, and instead of performing bulk estimates after all the data is collected, our estimation algorithm is executed each time a new observation appears. Furthermore, each estimate is obtained using only the 20 most current observations (i.e., $2 \mathrm{~s}$ of the most recent data). The results of this calculation are plotted in Fig. 7 for two different prior assumptions. The first, nominal prior case, assumes that the nominal coefficients are known to high precision ahead of 


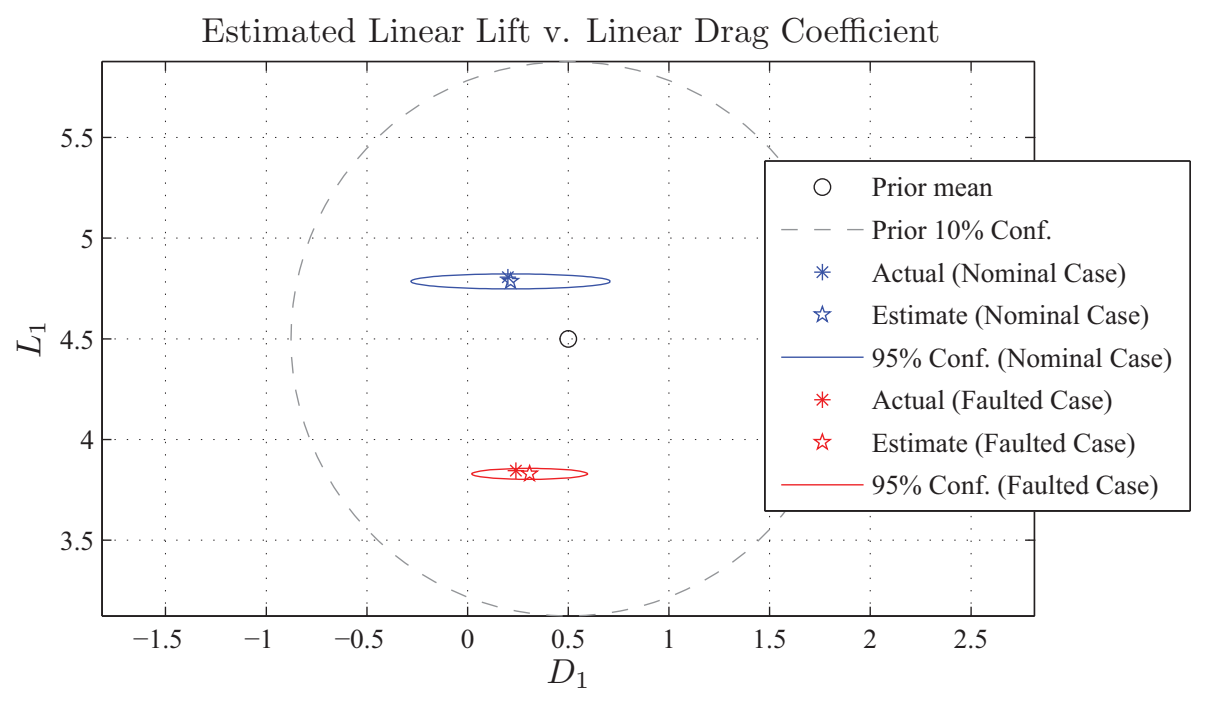

Figure 6: Estimation results from trajectory and accelerometer measurements for nominal and $20 \%$ fault models.

time, corresponding to the case where one expects the aircraft to behave normally. The second, open prior case, assumes the prior is a broad distribution on the model parameters, corresponding to an openness to learn the behavior of the aircraft from only its most recent maneuvering performance. These two cases are viewed as two separate modeling assumptions with different evidence calculations. At the onset of the fault, the evidence immediately collapses in both cases. The calculation then remains collapsed for the strong highprecision prior, because the measured data contradicts the prior assumption in this case. This is in contrast to the open prior case, which recovers the faulted aerodynamic coefficients and evidence level within the 20 sample window. The evidence collapse in each case is most significant at the onset of the fault, because in each case the new faulted data point contradicts the current state of knowledge given the past observations most strongly. To examine this phenomenon a little closer, the bottom plot shows a histogram of the change in evidence as each new measurement is added (and an old measurement is forgotten). The change caused by the fault is clearly several standard deviations from the natural variation in evidence for our small sample window, demonstrating a near instantaneous ability to detect the fault in this case.

\section{Maneuverability Beyond Trim}

Based on the aerodynamic information recovered through the system identification process discussed in $\S I I I$, and on the trim envelope calculated in $\S I I . B$, one is now able to obtain extended safe maneuvering envelopes by using the robust reachability analysis summarized in this section.

\section{IV.A. Interpretation of the Maneuvering Envelope}

In this context, the preferred interpretation of the safe maneuvering envelope considers reachability from the trim envelope. The stable and controllable trim envelope is considered an a-priori safe set. The backwards reachable set is defined as the set of states from where (at least one point in) the trim envelope can be reached. The forwards reachable set is defined as the set of states which can be reached from (at least one point in) the trim envelope. The safe maneuvering flight envelope is then the intersection between the forwards and backwards reachable sets. This interpretation is illustrated in Fig. 8. In addition to the safe envelope, the backwards reachable set is considered as the survivable flight envelope. After an upset due to 

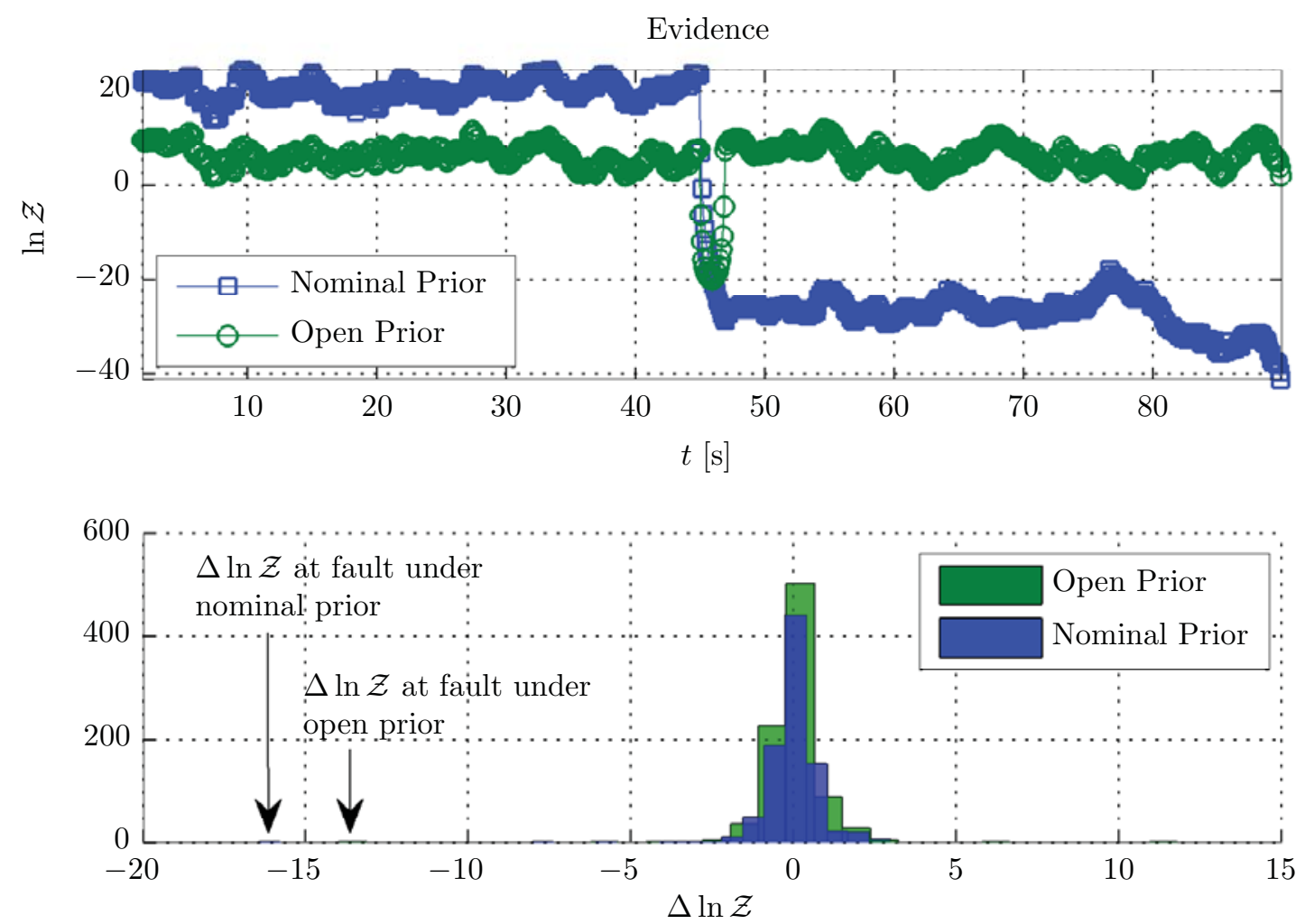

Figure 7: Fault identification from evidence. The top plot shows the running evidence calculation, for two separate prior assumptions, based on a 20 sample window of the most recent data. The bottom plot shows a histogram of the change in evidence as each new sample is added into the window (and the last sample is forgotten). Clearly, the change in evidence caused by the fault is significantly greater than the natural variation in evidence caused by new measurements. This enables near instantaneous identification of the fault in this scenario.

damage, turbulence, a wake encounter etc., it is possible to bring the aircraft back to a safe trim condition as long as the current flight condition is situated inside the backwards reachable set.

The goal, then, is to perform a combined forward and backward reachability analysis from the trim envelope as efficiently as possible, in an effort to enable online implementations. Based on previous research, ${ }^{37}$ level set methods are an excellent candidate. Finally, robustness to the uncertainty in the system identification (i.e., aerodynamic parameter estimates) is an important aspect to consider as well.

\section{IV.B. Optimal Control Formulation}

It has been shown in the literature that maneuvering envelope estimation through reachability can be reformulated in the optimal control framework. ${ }^{9}$ Consider our continuous time control system:

$$
\dot{x}=f\left(x, u ; c^{\star}+\Delta\right)
$$

with $(x, u) \in \mathcal{B}$, as defined in $\S I I . B$ above. Here we consider the case where the aerodynamic coefficient vector can take on any value within a probable set $\left\{c=c^{\star}+\Delta \mid \Delta \in D \subseteq \mathbb{R}^{d}\right\}$, such as a $95 \%$ confidence ellipse. Define $\phi(\tau, t, x, u(\cdot), \Delta)$ as the state trajectory, where $\Delta$ characterizes possible parameter uncertainty. Given a set of states $K \subseteq \mathbb{R}^{n}$, and a set of possible input functions $\mathcal{U}_{\left[t, t^{\prime}\right]}$, the reachability question is naturally 


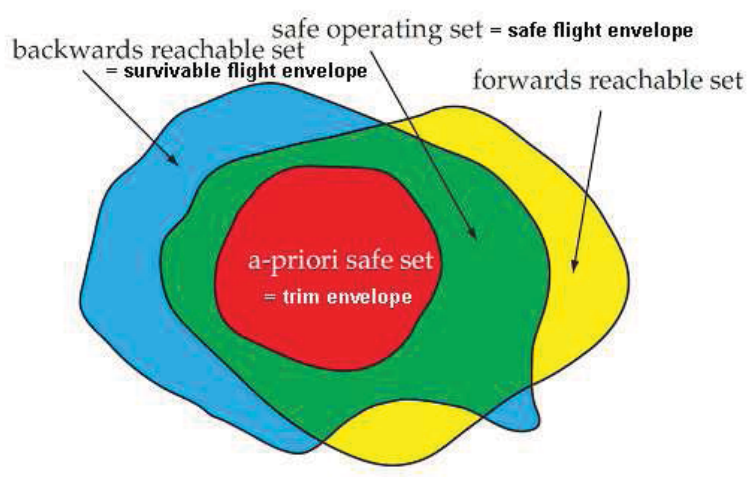

Figure 8: Safe maneuvering envelope as intersection between forwards and backwards reachability, modified from source: van Oort. ${ }^{12}$

formulated using the relation between the set $K$ and the state trajectories $\phi$ of (34) over the horizon $T$. The problem of interest is the following:

Robust reachability: Does there exist a $u \in \mathcal{U}_{[0, T]}$ and a $t \in[0, T]$ such that the trajectory $\phi$ of the state $x$ satisfies $x \in K$, irrespective of $\Delta$ ?

The answer to this question is found by playing a pursuit evasion game over the horizon $T \geq 0$ with target set $K \subseteq \mathbb{R}^{n}{ }^{38}$ It is assumed that $u$ is trying to bring or keep the state in the set $K$, whereas $\Delta$ is trying to drive it out of $K$. To ensure the game is well-posed, $u$ is restricted to play non-anticipative strategies with respect to the parameter uncertainty $\Delta \in D$.

For the types of safety problems considered here, a set of initial states has to be established such that $u$ can win the game. To this end, the robust reachable set $\mathcal{R}_{\Delta}$ is defined as,

$$
\mathcal{R}_{\Delta}(t, K)=\left\{x \in \mathbb{R}^{n} \mid \forall \Delta \in D, \exists u \in \mathcal{U}_{[t, T]}, \exists \tau \in[t, T], \phi(\tau, t, x, u(\cdot), \Delta) \in K\right\} .
$$

As shown in the literature, a useful characterization of this set is found by solving an INFMIN problem. ${ }^{9}$ The crux is to include the $\Delta$ 's as disturbances opposing the optimization over $u$. Consider a closed set $K$, defined as the level set of a continuous function $l: \mathbb{R}^{n} \rightarrow \mathbb{R}$, i.e., $K=\left\{x \in \mathbb{R}^{n} \mid l(x) \geq 0\right\}$. We then define the invariance set as ${ }^{11}$

$$
\operatorname{Inv}(t, K)=\left\{x \in \mathbb{R}^{n} \mid V_{2}(x, t) \geq 0\right\},
$$

where,

$$
V_{2}(x, t)=\inf _{u(\cdot) \in \mathcal{U}_{[t, T]}} \sup _{\Delta \in D} \min _{\tau \in[t, T]} l(\phi(\tau, t, x, u(\cdot), \Delta)) .
$$

The solution for $V_{2}$ is then found by solving the associated Hamilton-Jacobi-Bellman (HJB) Partial Differential Equation (PDE): $:^{9,38}$

$$
\frac{\partial V_{2}}{\partial t}(x, t)+\min _{\tau \in[t, T]}\left\{\inf _{u(\cdot) \in \mathcal{U}_{[t, T]}} \sup _{\Delta \in D} \frac{\partial V_{2}}{\partial x}(x, t) f(x, u, \Delta)\right\}=0,
$$

where $V_{2}(x, T)=l(x)$ holds for backward integration and $V_{2}(x, t)=l(x)$ applies to forward integration. These HJB PDE's can be solved using level set methods, for which a toolbox is available in MATLAB ${ }^{\circledR} .10$ Finally, the robust reachability set is found through duality: $\mathcal{R}_{\Delta}(t, K)=\left[\operatorname{Inv}\left(t, K^{c}\right)\right]^{c}$. See Ref. [39] for further details.

\section{IV.C. Examples}

The scenario to be considered is the $(V, \gamma)$ maneuvering envelope for the previously introduced RCAM model with bank angle $\varphi=0$. Both the nominal and generic damage scenarios will be evaluated. The damage 
scenario is the one considered previously in §II.C, with a $20 \%$ decrease (increase) in lift (drag) performance, as well as envelope degradation caused by $50 \%$ loss of maximum thrust authority. This example builds further on the results for the trim envelope presented in $\S$ II.C. The results are shown in Figs. 9-11. Backward reachability analyses corresponding to the survivable maneuvering envelope are presented in Fig. 9. Fig. 10 shows the forward reachability analysis results. The intersection of forwards and backwards reachability is defined as the safe maneuvering envelope and is shown in Fig. 11. The time horizon in this example is set at $T=5 \mathrm{~s}$. This choice is based on the time constants of the considered relevant dynamics. The blue rectangular contour corresponds to the largest rectangle which can be drawn in the trim envelope of the nominal aircraft as depicted in Fig. 4. Similarly the red contours correspond to the largest rectangles which can be spanned in the trim envelopes of both damage scenarios in Fig. 4.

Fig. 9 analyzes backward reachability from the trim envelopes over a time horizon of $T=5 \mathrm{~s}$. By comparing the undamaged and damaged envelope boundaries, one can see the influence of the damage characteristics. It can be seen that for lower speed conditions, recovery with damage is only possible for a more restricted range of negative flight path angles. The increased drag results in a higher minimum as well as maximum airspeed limit, since accelerating works slower and decelerating works faster. The loss in lift capability means that smaller positive flight path angles are backwards reachable at lower airspeed. The scenario with the reduced maximum thrust limit is similar, but negative flight path angles at lower speeds are even more restricted due to the extra limitation in thrust. Furthermore, the reduction in backwards reachable positive flight path angles is more significantly influenced by the reduced trim envelope, than by the actual reachability calculations over $5 \mathrm{~s}$.

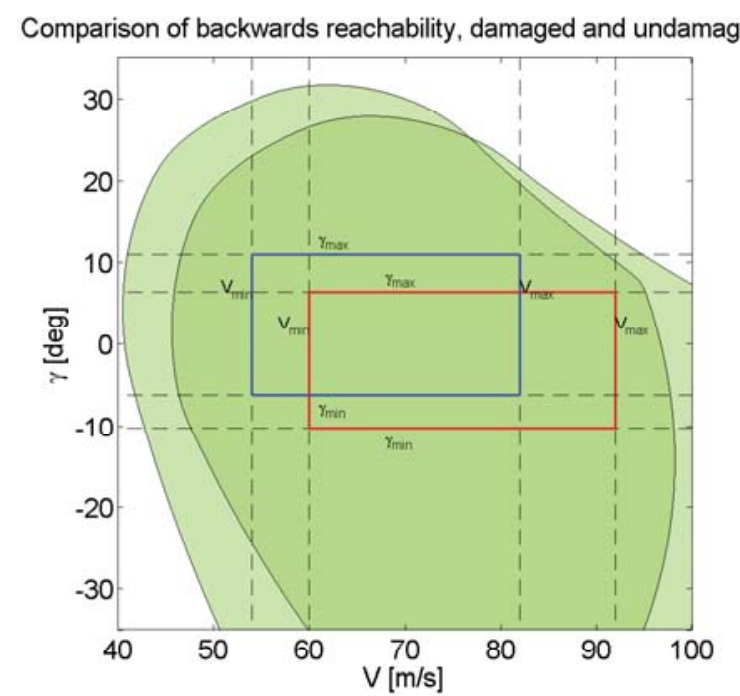

(a) Effect of a $20 \%$ decrease in the lift coefficients, and a $20 \%$ increase in the drag coefficients

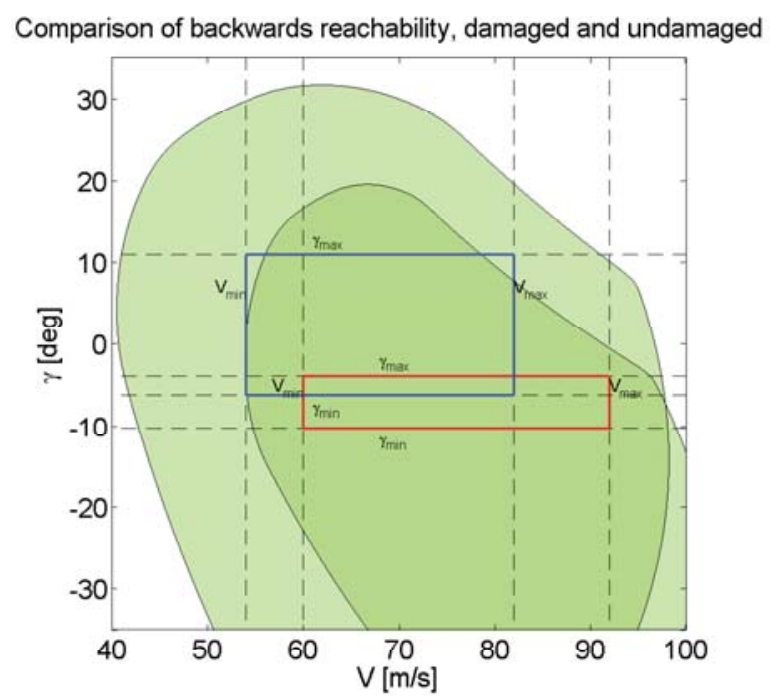

(b) Additional envelope degradation caused by $50 \%$ loss of maximum thrust authority

Figure 9: Backward reachability analysis over $T=5 \mathrm{~s}$, based on calculated trim envelope boundaries and identified aerodynamic parameters. Upper left envelope areas correspond to nominal configuration.

Fig. 10 shows the forward reachable set calculations over the same time horizon. One may observe that there is less range across larger flight path angle values in the damage scenarios due to the reduction in lift capability. Fig. 10(a) shows that larger positive flight path angle values are still reachable at higher speeds with damage. This is related to the fact that the trim envelope of the damaged scenario has moved towards a higher airspeed range, which makes comparing both envelopes less straightforward. Forwards reachability for the scenario depicted in Fig. 10(b) is similar, but forwards reachability for positive flight path angles is further reduced due to the changed trim envelope boundaries and the extra limitation on thrust. Comparing Fig. 10(a) and 10(b), one finds that the reduced thrust authority also affects the reachable speed range, which is now more restricted at the upper and lower boundaries. 


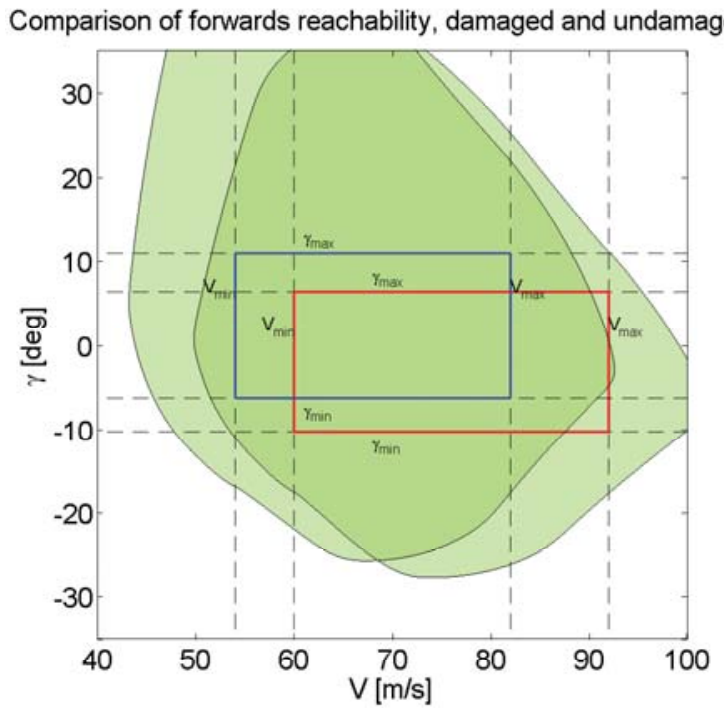

(a) Effect of a $20 \%$ decrease in the lift coefficients, and a $20 \%$ increase in the drag coefficients

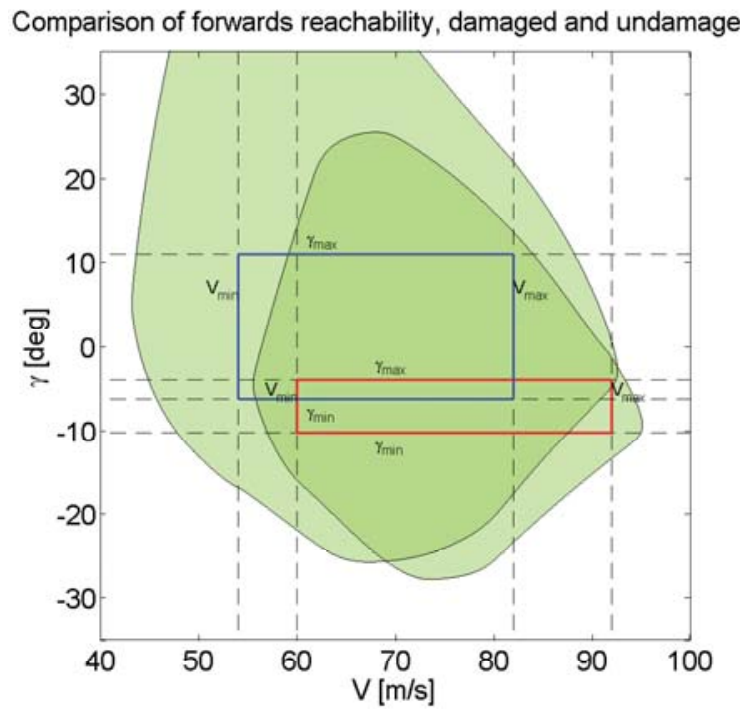

(b) Additional envelope degradation caused by $50 \%$ loss of maximum thrust authority

Figure 10: Forward reachability analysis over $T=5 \mathrm{~s}$, based on calculated trim envelope boundaries and identified aerodynamic parameters. Upper left envelope areas correspond to nominal configuration.

The safe maneuvering envelopes presented in Fig. 11 are the result of taking the intersection between the backward and forward reachability envelopes in Figs. 9 and 10. In Fig. 11(a), it can be seen that the safe maneuvering envelope shift is similar in nature to the trim envelope shift shown in Fig. 4. A higher speed range is needed to compensate for the loss in the lift force coefficient, and smaller positive flight path angles can be reached because smaller excess of net thrust is available with increased drag. In Fig. 11(b), one may observe that the restriction on maximum thrust leads to a further shrinking of the maneuvering envelope, especially for positive flight path angles. Level and climbing flight are still possible, but only in non-trim and as a trade-off between kinetic and potential energy. One has to sacrifice speed for non-negative flight path angles. In practice, however, it is the other way around: one would rather sacrifice flight path angle for airspeed, in order to prevent stalling.

Extensive Monte Carlo analyses were also performed in order to verify the accuracy of the boundaries of the estimated maneuvering envelopes. These analyses were based on the non-simplified aircraft model, ignoring the assumption that the aerodynamic angles $\alpha$ and $\beta$ should be small. All these Monte Carlo analyses have confirmed that the results provided here are accurate and that the simplifications hold for the current ranges of the aerodynamic angles, namely $\alpha \in\left[0^{\circ} ; 14.5^{\circ}\right]$ (no stall) and $\beta \in\left[-5^{\circ} ;+5^{\circ}\right]$. This is an important conclusion which makes a relevant online safe maneuvering envelope estimation tool much more feasible. This result is covered in [39], along with more examples and discussion.

\section{Major Application Areas}

\section{V.A. Flight Management Systems}

The Flight Management System (FMS) of commercial aircraft is a common tool to aid crew in piloting aircraft. The FMS is comprised of many functions, including navigation, flight planning, trajectory generation, performance computations, and guidance. With the exception of navigation, all these capabilities are either directly or indirectly dependent on aircraft performance characteristics; and when these characteristics change due to vehicle impairment, the ability of the aircraft to satisfy the constraints of the existing flight plan or to follow the recommended trajectory, guidance commands, or performance profiles is compro- 


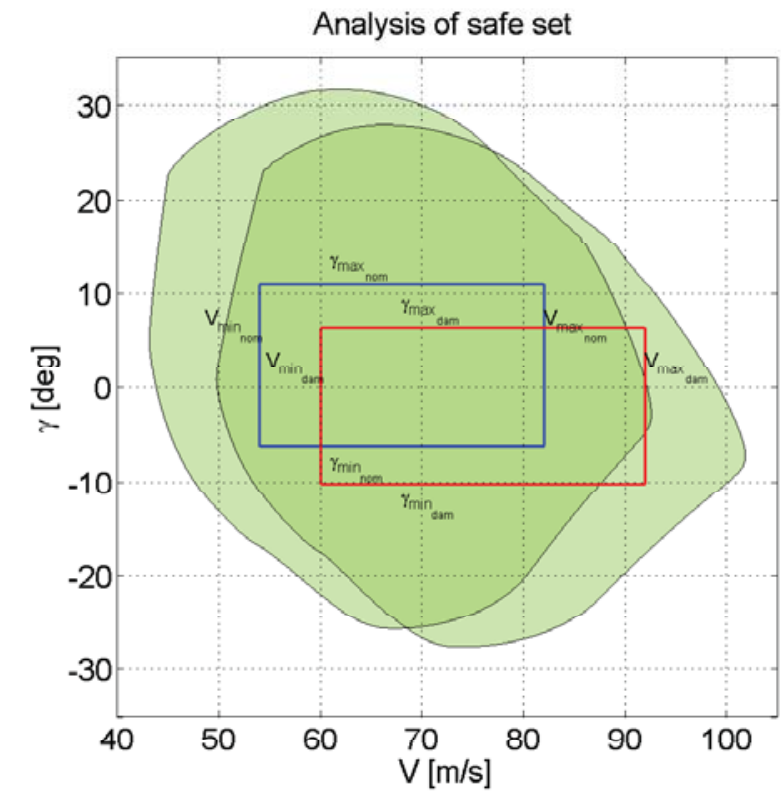

(a) Effect of $20 \%$ decrease in lift coefficient and $20 \%$ increase in drag coefficient

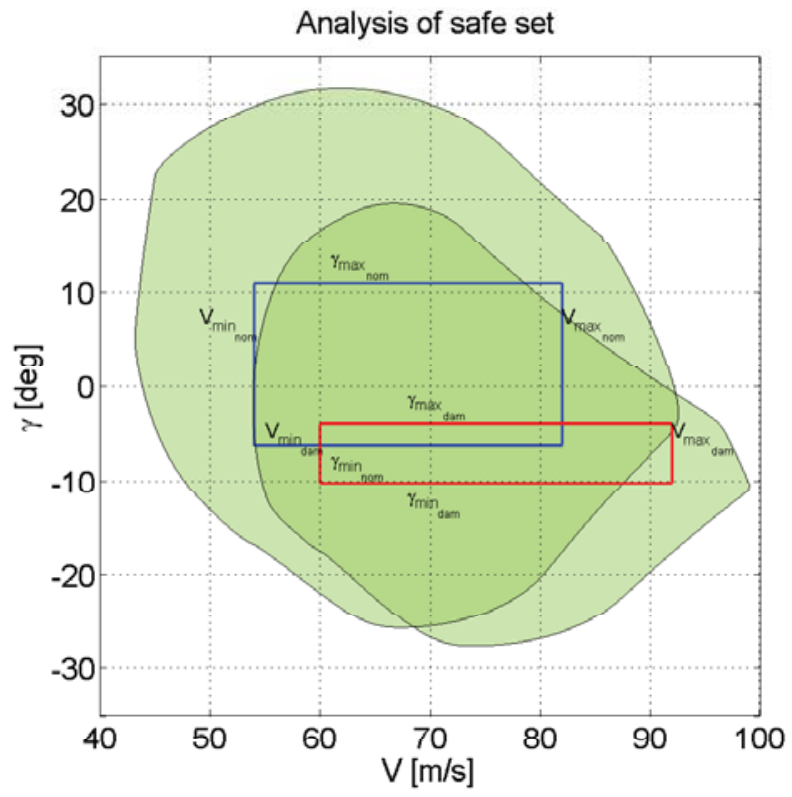

(b) Additional envelope degradation caused by $50 \%$ loss of maximum thrust authority

Figure 11: Calculation of safe maneuvering envelope sets through forward and backward reachability analysis over $T=5 \mathrm{~s}$, based on calculated trim envelope boundaries and identified aerodynamic parameters. Upper left envelope areas correspond to nominal configuration.

mised. Therefore, the trim and maneuverability envelope calculations discussed in this paper enable envelope protection enhancements for next generation flight management systems, like the one discussed in [40].

\section{V.B. Pilot Cockpit Displays}

Pilot cockpit displays are instrumental for pilot situational awareness of aircraft state and performance limitations. Performance limitations such as V-speeds or maximum and minimum speeds for the nominal configuration are currently determined from performance databases that will become obsolete in the event of failure, damage, or impairment conditions. Even under nominal conditions, the relationship between maneuverable airspeed and vertical speed changes in non-obvious ways with altitude, air density, aircraft configuration (e.g., flap settings, stabilizer position), and bank angle. A recent survey of airline transport rated pilots demonstrated a preference for the display of maneuvering limitations in bank angle, vertical velocity, and aircraft speed, ${ }^{41}$ all of which are readily provided by the envelope characterization methods discussed here.

\section{Conclusion}

There are three distinguishing features of the maneuverability characterization methods presented in this article.

First, instead of linearizing the full aircraft dynamics model, we derived a useful nonlinear model that allowed us to avoid local linear approximations all together. This enabled rapid numerical computation of the curved (non-convex) trim envelope, which is more representative of the global aircraft performance capability than would be obtained through the use of linearized models.

Second, our integrated system identification approach leverages the same dynamics model to estimate the underlying aerodynamic parameters from currently available flight data, while also establishing confidence 
regions biased towards greater uncertainty when insufficient input excitation is available. This is important because the penalty for over-confidence in-flight can be severe, while the cost of under-confidence is often only over-preparedness and caution. Furthermore, the system identification process seamlessly provides methods for learning the aircraft dynamics passively in the background, detecting impairment, and also selecting between multiple nonlinear dynamics models for damaged aircraft through the calculation of Bayesian evidence.

Third, through the safe maneuvering envelope calculation, we effectively maximized the options pilots or automation systems have to recover the aircraft in damage scenarios, while remaining robust to the uncertainty in the system identification process.

The net result is an increased flexibility for developing advanced aircraft diagnostics that provide the bottom line maneuverability of the aircraft as an output, and this is expected to have important applications to flight planning, trajectory generation, guidance algorithms, and pilot displays.

\section{Acknowledgments}

This work is supported by NASA's Aviation Safety Program, through the Vehicle Systems Safety Technologies (VSST) project, and by a Marie Curie International Outgoing Fellowship (IOF) within the 7th European Community Framework Program.

\section{References}

${ }^{1}$ Yunshen Tang, Ella M. Atkins, and Robert M. Sanner. Emergency flight planning for a generalized transport aircraft with left wing damage. In Proceedings of the AIAA Guidance, Navigation and Control Conference, number AIAA 2007-6873, August 2007.

${ }^{2}$ N. Meuleau, C. Plaunt, D. Smith, and T. Smith. A comparison of risk sensitive path planning methods for aircraft emergency landing. In ICAPS-09 Workshop on Bridging the Gap Between Task and Motion Planning, 2009.

${ }^{3}$ N. Meuleau, C. Neukom, C. Plaunt, D. Smith, and T. Smith. The emergency landing planner experiment. In ICAPS-11 Scheduling and Planning Applications Workshop, 2011.

${ }^{4}$ Thomas Lombaerts. Fault Tolerant Flight Control - A Physical Model Approach. Phd dissertation, Delft University of Technology, May 2010. Available at: http://repository.tudelft.nl/view/ir/uuid\%3A538b0174-fe84-43af-954d-02f256b2ec50/.

${ }^{5}$ Liang Tang, Michael Roemer, Sudarshan Bharadwaj, and Christine Belcastro. An integrated health assessment and fault contingency management system for aircraft. In AIAA Guidance, Navigation and Control Conference, number AIAA 2008-6505, August 2008.

${ }^{6}$ M.G. Goman, A.V. Khramtsovsky, and E.N. Kolesnikov. Evaluation of aircraft performance and maneuverability by computation of attainable equilibrium sets. Journal of Guidance, Control and Dynamics, 31(2):329-339, March - April 2008.

${ }^{7}$ Jeffrey D. Keller, Robert M. McKillip, and Sungwan Kim. Aircraft flight envelope determination using upset detection and physical modeling methods. In AIAA Guidance, Navigation and Control Conference, number AIAA 2009-6256, August 2009.

${ }^{8}$ Jovan D. Boskovic, Joshua Redding, and Nathan Knoebel. An adaptive fault management (afm) system for resilient flight control. In AIAA Guidance, Navigation and Control Conference, number AIAA 2009-6263, August 2009.

${ }^{9}$ John Lygeros. On reachability and minimum cost optimal control. Automatica, 40:917-927, 2004.

${ }^{10}$ Ian M. Mitchell. The flexible, extensible and efficient toolbox of level set methods. Journal of Scientific Computing, 35:300-329, 2008.

${ }^{11}$ Ioannis Kitsios and John Lygeros. Launch pad abort flight envelope computation for a personnel launch vehicle using reachability. In AIAA Guidance, Control and Navigation, number AIAA 2005-6150, August 2005.

${ }^{12}$ Eddy Van Oort, Ping Chu, and Jan Albert Mulder. Advances in Aerospace Guidance, Navigation and Control, chapter Maneuvering Envelope Determination through Reachability Analysis, pages 91-102. Springer - Verlag, 2011.

${ }^{13}$ Shahab Kaynama, John Maidens, Meeko Oishi, Ian M. Mitchell, and Guy A. Dumont. Computing the viability kernel using maximal reachable sets. In Proceedings of the 15th ACM International Conference on Hybrid Systems: Computation and Control, HSCC '12, pages 55-64, New York, NY, USA, 2012. ACM.

${ }^{14}$ Rohit Pandita, Abhijit Chakraborty, Peter Seiler, and Gary Balas. Reachability and region of attraction analysis applied to gtm dynamic flight envelope assessment. In AIAA Guidance, Navigation and Control Conference, number AIAA 2009-6258, August 2009

${ }^{15}$ Barton J. Bacon. Quaternion based control architecture for determining controllcontrol/maneuverability limits. In Guidance, Navigation, and Control and Co-located Conferences, number AIAA 2012-5028. American Institute of Aeronautics and Astronautics, August 2012.

${ }^{16}$ Jong-Yeob Shin and Christine Belcastro. Robustness analysis and reliable flight regime estimation of an integrated 
resilient control system for a transport aircraft. In AIAA Guidance, Navigation and Control Conference, number AIAA 20086656, August 2008.

${ }^{17}$ Matthew D. Lichter, Alec J. Bateman, and Gary Balas. Flight test evaluation of a rum-time stability margin estimation tool. In AIAA Guidance, Navigation and Control Conference, number AIAA 2009-6257, August 2009.

${ }^{18}$ James M. Urnes, Eric Y. Reichenbach, and Timothy A. Smith. Dynamic flight envelope assessment and prediction. In AIAA Guidance Navigaton and Control Conference, number AIAA 2008-6983, August 2008.

${ }^{19}$ Mario G. Perhinschi, Hever Moncayo, and Jennifer Davis. Integrated framework for aircraft sub-system failure detection, identification and evaluation based on the artificial immune system paradigm. In AIAA Guidance, Navigation and Control Conference, number AIAA 2009-6261, August 2009.

${ }^{20}$ Sebastian P. Sanchez, Mario G. Perhinschi, Hever Moncayo, Marcello R. Napolitano, Jennifer Davis, and Mario L. Fravolini. In-flight actuator failure detection and identification for a reduced size uav using the artificial immune system approach. In AIAA Guidance Navigation and Control Conference, number AIAA 2009-6266, August 2009.

${ }^{21}$ Hever Moncayo, Mario Perhinschi, and Jennifer Davis. Artificial immune system - based aircraft failure evaluation over extended flight envelope. In Guidance, Navigation, and Control and Co-located Conferences, number AIAA $2010-8038$. American Institute of Aeronautics and Astronautics, August 2010.

${ }^{22}$ Agostino De Marco, Eugene L. Duke, and Jon S. Berndt. A general solution to the aircraft trim problem. In AIAA Modeling and Simulation Technologies Conference, number AIAA 2007-6703, August 2007.

${ }^{23}$ Guoxing Yi and E.M. Atkins. Methods of trim state discovery. In Systems and Control in Aeronautics and Astronautics (ISSCAA), 2010 3rd International Symposium on, pages 654-659, june 2010.

${ }^{24}$ Shankar Sastry. Nonlinear Systems: Analysis, Stability, and Control. Springer-Verlag, 1999.

${ }^{25}$ Gertjan Looye and Samir Bennani. Description and analysis of the research civil aircraft model (rcam). Technical report TP-088-27, Group for Aeronautical Research and Technology in Europe GARTEUR, 1997.

${ }^{26}$ Eugene A. Morelli. Efficient global aerodynamic modeling from flight data. In 50th AIAA Aerospace Sciences Meeting, number AIAA 2012-1050, 2012.

${ }^{27}$ T.J.J. Lombaerts, E.R. Van Oort, J.A. Mulder Q.P. Chu, and D.A. Joosten. Online aerodynamic model structure selection and parameter estimation for fault-tolerant control. Journal of Guidance, Control, and Dynamics, 33(3):707-723, May-June 2010.

${ }^{28}$ Padmanabhan K. Menon, Prasenjit Sengupta, Sai Vaddi, Bong-Jun Yang, and Jason Kwan. Impaired aircraft performance envelope estimation. Journal of Aircraft, 50(2):410-424, March-April 2013.

${ }^{29}$ Vladislav Klein and Eugene A. Morelli. Aircraft System Identification, Theory and Practice. American Institute of Aeronautics and Astronautics, Inc., 2006.

${ }^{30}$ Eugene A. Morelli and Kevin Cunningham. Aircraft dynamic modeling in turbulence. In AIAA Atmospheric Flight Mechanics Conference, number AIAA 2012-4650. AIAA, August 2012.

${ }^{31}$ Eugene A. Morelli, Kevin Cunningham, and Melissa A. Hill. Global aerodynamic modeling for stall/upset recovery training using efficient piloted flight test techniques. In AIAA Modeling and Simulation Technologies (MST) Conference, number AIAA 2013-4976, December 2013.

${ }^{32}$ V. N. Smelyanskiy, D. G. Luchinsky, D. A. Timuçin, and A. Bandrivskyy. Reconstruction of stochastic nonlinear dynamical models from trajectory measurements. Phys. Rev. E, 72:026202, Aug 2005.

${ }^{33}$ D. Bertsekas. Nonlinear Programming. Athena Scientific, Cambridge, MA, 2nd edition, 1999.

${ }^{34} \mathrm{~S}$. Boyd and L. Vandenberghe. Convex Optimization. Cambridge University Press, Cambridge, UK, 2004.

${ }^{35}$ A. Gelman, J. Carlin, H. Stern, and D. Rubin. Bayesian Data Analysis. Chapman \& Hall/CRC, 2nd edition, 2004.

${ }^{36}$ D.S. Sivia and D. Skilling. Data Analysis, A Bayesian Tutorial. Oxford University Press, 2nd edition, 2006.

${ }^{37}$ Nithin Govindarajan. An optimal control approach for estimating aircraft command margin - with applications to loss-of-control prevention. Masters thesis, Delft University of Technology, October $2012 . \quad$ Available at: http://repository.tudelft.nl/view/ir/uuid\%3A48dbad61-728a-4c7e-ba3e-cf8382ef1cef/.

${ }^{38}$ Ioannis Kitsios and John Lygeros. Final glide-back envelope computation for reusable launch vehicle using reachability. In IEEE Conference on Decision and Control, and the European Control Conference, pages pp. 4059 - 4064, December 2005.

${ }^{39}$ Thomas Lombaerts, Stefan Schuet, Kevin Wheeler, Diana Acosta, and John Kaneshige. Safe maneuvering envelope estimation based on a physical approach. In AIAA Guidance, Navigation and Control Conference, number AIAA 2013-4618, August 2013

${ }^{40}$ John Kaneshige, Ramesh Panda, Gordon Hardy, Mieczyslaw Steglinski, Shivanjli Sharma, and Jose Benvides. Tactical flight management concept for trajectory based operations. In Proceedings of the AIAA Infotech@Aerospace Conference, number AIAA 2012-2558, June 2012.

${ }^{41}$ Anna Trujillo and Irene Gregory. Pilot preferences on displayed aircraft control variables. In Don Harris, editor, Engineering Psychology and Cognitive Ergonomics. Applications and Services, volume 8020 of Lecture Notes in Computer Science, pages 193-202. Springer Berlin Heidelberg, 2013. 